\title{
Interaction of Rarefaction Waves of the Two-Dimensional Self-Similar Euler Equations
}

\author{
Jiequan $\mathrm{Li}^{1}$ \\ Department of Mathematics, Capital Normal University, Beijing, 100037 \\ Yuxi Zheng ${ }^{2}$ \\ Department of Mathematics, The Pennsylvania State University, PA 16802
}

November 10, 2006

\begin{abstract}
Classical self-similar solutions to the interaction of two arbitrary planar rarefaction waves for the polytropic Euler equations in two space dimensions are constructed. The binary interaction represents a major type of interaction in the two-dimensional Riemann problems, and includes in particular the classical problem of the expansion of a wedge of gas into vacuum. Based on the hodograph transformation, the method involves the phase space analysis of a second-order equation and the inversion back to (or development onto) the physical space.
\end{abstract}

Keywords: Simple waves, hodograph transformation, Jacobian, characteristic decomposition, inclination angles of characteristics, 2-D Riemann problem, compressible, gas dynamics.

AMS subject classification: Primary: 35L65, 35J70, 35R35; Secondary: 35J65.

\section{Introduction}

Consider the two-dimensional isentropic compressible Euler system

$$
\left\{\begin{array}{l}
\rho_{t}+(\rho u)_{x}+(\rho v)_{y}=0 \\
(\rho u)_{t}+\left(\rho u^{2}+p\right)_{x}+(\rho u v)_{y}=0 \\
(\rho v)_{t}+(\rho u v)_{x}+\left(\rho v^{2}+p\right)_{y}=0
\end{array}\right.
$$

\footnotetext{
${ }^{1}$ Research partially supported by NSF from Beijing Municipality and the Key Program from Beijing Educational Commission with no. KZ200510028018, Program for New Century Excellent Talents in University (NCET) and Funding Project for Academic Human Resources Development in Institutions of Higher Learning Under the Jurisdiction of Beijing Municipality (PHR-IHLB).

${ }^{2}$ Research partially supported by NSF-DMS-0305497, 0305114, 0603859
} 
where $\rho$ is the density, $(u, v)$ is the velocity and $p$ is the pressure given by $p(\rho)=K \rho^{\gamma}$ where $K>0$ will be scaled to be one and $\gamma>1$ is the gas constant. We are primarily interested in the so-called pseudo-steady case of (1.1); i.e., the solutions depend on the self-similar variables $(\xi, \eta)=(x / t, y / t)$. The expansion of a wedge of gas into vacuum is such a case. Assuming the flow is irrotational, a classical hodograph transformation (see [21]) can be used to eliminate the two self-similar variables to result in a partial differential equation of second order for the speed of sound $c$ in the velocity variables $(u, v)$. It has been known that the difficulties of the procedure are that the transformation is degenerate for common waves such as the constant states and some other types of waves resembling the simple waves of the steady Euler system, and the transformation of boundaries is difficult to handle. In 2001, Li ([13]) carried out an anaysis of the second order equation in the space $(c, u, v)$, where he discovered a pair of variables resembling the well-known Riemann invariants together with their invariant regions and established the existence of a solution to the expansion of a wedge of gas into vacuum in the hodograph plane for wide ranges of the gas constant and the wedge angle. Recently in 2006, paper [15], in an attempt to establish the inversion of the hodograph mapping, clarified the concept of simple waves for (1.1). We show in this paper that the hodograph transformation is non-degenerate precisely for non-simple waves, and all the solutions constructed in [13] in the hodograph plane can now be transformed back to the self-similar plane. Thus we complete the procedural circle of construction of solutions.

We find that the circle of construction bears very interesting similarity to the construction of centered rarefaction waves in the one-dimensional systems of conservation laws. The selfsimilar variable(s) in both cases decouple from the phase space(s), and the equations in the phase space(s) are solved first. The development of the solutions from the phase space onto the physical space(s) requires genuine nonlinearity in the one-dimensional case and a nondegeneracy condition of the hodograph transformation in the two-dimensional case.

The key ingredient of the paper is the simplification of the form of the equations in the phase space brought about by the employment of the inclination angles of characteristics. The approach now presents itself as a method of significant potential for the study of the pseudo-steady Euler system in hyperbolic regions. It yields structure of solutions in addition to existence. We use this approach for example to establish the Lipschitz continuity and monotonicity behavior of the vacuum boundary in the problem of a wedge of gas into vacuum and establish the dependence of the location of the boundary on the wedge angle and gas constant. The approach is particularly suitable for studying two-dimensional Riemann problems ([26]), since the apparent nature of the solutions of a Riemann problem is piece-wise smooth.

The expansion problem of gas into vacuum has been a favorite for a long time. The problem has been interpreted hydraulically as the collapse of a wedge-shaped dam containing water initially with a uniform velocity, see Levine [11]. In Suchkov [24], a set of interesting explicit solutions were found. Mackie [19] proposed a scalar equation of second order for a potential function, studied the interface of gas and vacuum by the method of unsteady Prandtl-Meyer expansions and related it to the PSI approach in [21], from which Li ([13]) started with new motivation from the success on the pressure gradient system ([7]). In the context of two- 
dimensional Riemann problems, the expansion problem of a wedge of gas into vacuum is the interaction of two two-dimensional planar rarefaction waves. We see it as one of two possible interactions of continuous waves in the hyperbolic region; this one expands without shocks but with a boundary degeneracy, while the other one forms shocks with a sonic boundary as well as a shock boundary. The method applies locally in both cases. A quick round-up of cases that involves hyperbolic regions of non-constant continuous waves [1, 2, 10, 3, 8, 22, 14, 27] show that the approach taken here has general applicability.

Our main results are the simple form of the equations in the phase space $(c, u, v)$ (system (6.16)), the existence of solutions of the expansion of a wedge of gas into vacuum (Theorem 7.2), and the detailed properties of the expansion (Theorems 7.3-7.4). We provide some background information as well regarding the hodograph transformation and simple waves in Sections 2-5 for the convenience of nonexpert readers. Section 6 is for the phase space analysis, while Section 7 handles the gas expansion problem. We point out that the main work of this paper is the estimates of the solutions in the phase space for establishing the validity of the inversion of the hodograph transformation. The notorious difficulty of the inversion manifests itself in the fact that the characteristics in the phase space do not have a fixed concavity type although the corresponding characteristics in the physical space do, see Subsections 7.5 and 7.7. We mention additionally that hodograph transforms have been used in various forms, see [20,4] and references therein.

Here is a list of our notations: $\rho$ density, $p$ pressure, $(u, v)$ velocity, $c=\sqrt{\gamma p / \rho}$ speed of sound, $i=c^{2} /(\gamma-1)$ enthalpy, $\gamma$ gas constant, $(\xi, \eta)=(x / t, y / t)$ the self-similar (or pseudosteady) variables, $\varphi$ pseudo-velocity potential, $\theta$ wedge half-angle, and

$$
U=u-\xi, V=v-\eta, \kappa=(\gamma-1) / 2, m=(3-\gamma) /(\gamma+1) .
$$

Letters $C, C_{1}$ and $C_{2}$ denote generic constants.

\section{Primary system}

Our primary system is system $(1.1)$ in the self-similar variables $(\xi, \eta)=(x / t, y / t)$ :

$$
\left\{\begin{array}{l}
(u-\xi) i_{\xi}+(v-\eta) i_{\eta}+2 \kappa i\left(u_{\xi}+v_{\eta}\right)=0, \\
(u-\xi) u_{\xi}+(v-\eta) u_{\eta}+i_{\xi}=0 \\
(u-\xi) v_{\xi}+(v-\eta) v_{\eta}+i_{\eta}=0
\end{array}\right.
$$

We assume further that the flow is ir-rotational:

$$
u_{\eta}=v_{\xi}
$$

Then, we insert the second and third equations of (2.1) into the first one to deduce the system,

$$
\left\{\begin{array}{l}
\left(2 \kappa i-(u-\xi)^{2}\right) u_{\xi}-(u-\xi)(v-\eta)\left(u_{\eta}+v_{\xi}\right)+\left(2 \kappa i-(v-\eta)^{2}\right) v_{\eta}=0 \\
u_{\eta}-v_{\xi}=0
\end{array}\right.
$$


supplemented by Bernoulli's law

$$
i+\frac{1}{2}\left((u-\xi)^{2}+(v-\eta)^{2}\right)=-\varphi, \quad \varphi_{\xi}=u-\xi, \varphi_{\eta}=v-\eta
$$

We remark that the difference between the pseudo-steady flow (2.3) and the steady case (3.27) (see Subsection 3.1) is that the latter is self-contained, since the sound speed $c$ can be expressed by a pointwise function of the velocity explicitly through Bernoulli's law (3.28).

\section{Concept of Hodograph Transformation}

We introduce briefly the well-known hodograph transformation. The original form of a hodograph transformation is for a homogeneous quasi-linear system of two first-order equations for two known variables $(u, v)$ in two independent variables $(x, y)$. By regarding $(x, y)$ as functions of $(u, v)$ and assuming that the Jacobian does not vanish nor is infinity, one can re-write the system for the unknowns $(x, y)$ in the variables $(u, v)$, which is a linear system if the coefficients of the original system do not depend on $(x, y)$. See the book of Courant and Friedrichs [5]. Specifically, consider the system of two equations of the form,

$$
\left(\begin{array}{l}
u \\
v
\end{array}\right)_{x}+A(u, v ; x, y)\left(\begin{array}{l}
u \\
v
\end{array}\right)_{y}=0,
$$

where the coefficient matrix $A(u, v ; x, y)$ is

$$
A(u, v ; x, y)=\left(\begin{array}{ll}
a_{11} & a_{12} \\
a_{21} & a_{22}
\end{array}\right) .
$$

The two eigenvalues, denoted by $\Lambda_{ \pm}$, satisfies,

$$
\Lambda_{ \pm}^{2}-\left(a_{11}+a_{22}\right) \Lambda_{ \pm}+|A|=0 .
$$

We introduce the hodograph transformation,

$$
T:(x, y) \rightarrow(u, v) .
$$

Then (3.1) is reduced to the system

$$
\left(\begin{array}{c}
y_{v} \\
-y_{u}
\end{array}\right)+A(u, v ; x, y)\left(\begin{array}{c}
-x_{v} \\
x_{u}
\end{array}\right)=0 .
$$

Its eigenvalues, denoted by $\lambda_{ \pm}$, satisfy

$$
a_{12} \lambda_{ \pm}^{2}-\left(a_{22}-a_{11}\right) \lambda_{ \pm}-a_{21}=0 .
$$

Obviously, if the coefficient matrix $A$ does not depend on $(x, y),(3.5)$ becomes a linear system for the unknowns $(x, y)$.

The following proposition establishes the invariance of characteristics under the hodograph transformation. 
Proposition 3.1 (Invariance of characteristics). A characteristic of $(3.1)$ in the $(x, y)$ plane is mapped into a characteristic of (3.5) in the $(u, v)$ plane by the hodograph transform $T$.

Proof. Let $y=y(x)$ be a characteristic with $\frac{d y}{d x}=\Lambda_{ \pm}$. Its image is $v=v(u)$ under the hodograph transform (3.4). Then we have

$$
\frac{d y}{d x}=\frac{y_{u}+y_{v} \cdot \frac{d v}{d u}}{x_{u}+x_{v} \cdot \frac{d v}{d u}}=\Lambda_{ \pm}
$$

i.e.,

$$
\frac{d v}{d u}=-\frac{\Lambda_{ \pm} x_{u}-y_{u}}{\Lambda_{ \pm} x_{v}-y_{v}}
$$

Using (3.5) we find

$$
\left(\Lambda_{ \pm} x_{u}-y_{u}\right)+\lambda_{ \pm}\left(\Lambda_{ \pm} x_{v}-y_{v}\right)=0
$$

Therefore we have

$$
\frac{d v}{d u}=\lambda_{ \pm}
$$

which completes the proof of this proposition.

The idea of hodograph transformation does not obviously generalize to other systems such as system (2.3) of more than two simple equations or for inhomogeneous systems.

For (2.3), one realizes that the three variables $(i, u, v)$ are functions of $(\xi, \eta)$, so one can still try to use $(u, v)$ as the independent variables and regard $(\xi, \eta)$ as functions of $(u, v)$ and ultimately regard $i$ as a function of $(u, v)$. In this way we may obtain an equation for $i=i(u, v)$ in the plane $(u, v)$ which eliminates $(\xi, \eta)$. This was done in 1958 in a paper [21] by Pogodin, Suchkov and Ianenko, and has been referred to as the PSI approach. The implementation is as follows. Let the hodograph transformation be

$$
T:(\xi, \eta) \rightarrow(u, v)
$$

for (2.1), reverse the roles of $(\xi, \eta)$ and $(u, v)$ and regard $i$ as a function of $(u, v)$. Then $i$ as the function of $u$ and $v$ satisfies

$$
\left(u_{\xi} v_{\eta}-u_{\eta} v_{\xi}\right) d i=\left(i_{\xi} v_{\eta}-i_{\eta} v_{\xi}\right) d u+\left(-i_{\xi} u_{\eta}+i_{\eta} u_{\xi}\right) d v .
$$

We insert this into the law of momentum conservation of (2.1) and use the ir-rotationality condition (2.2) to obtain

$$
\begin{aligned}
& \xi-u=i_{u}, \\
& \eta-v=i_{v} .
\end{aligned}
$$

These interesting identities provide an explicit correspondence between the physical plane and the hodograph plane provided that the transformation $T$ is not degenerate. 
Therefore, using (3.13), we convert (2.3) into a "linear" (in fact, linearly degenerate, see Section 6) system

$$
\left\{\begin{array}{l}
\left(2 \kappa i(u, v)-i_{u}^{2}\right) \eta_{v}+i_{u} i_{v}\left(\xi_{v}+\eta_{u}\right)+\left(2 \kappa i-i_{v}^{2}\right) \xi_{u}=0 \\
\xi_{v}-\eta_{u}=0
\end{array}\right.
$$

for the unknowns $(\xi, \eta)$. The difficulty here is that $i$, as a function of $u$ and $v$, cannot be determined explicitly and point-wise. We will remark more later in contrast with the steady case.

We continue to differentiate (3.13) with respect to $u$ and $v$ :

$$
\begin{aligned}
\xi_{u}=1+i_{u u}, & \xi_{v}=i_{u v}, \\
\eta_{u}=i_{u v}, & \eta_{v}=1+i_{v v},
\end{aligned}
$$

and inserting these into the first equations of (3.14) to obtain,

$$
\left(2 \kappa i-i_{u}^{2}\right) i_{v v}+2 i_{u} i_{v} i_{u v}+\left(2 \kappa i-i_{v}^{2}\right) i_{u u}=i_{u}^{2}+i_{v}^{2}-4 \kappa i .
$$

This is a very interesting second order partial differential equation for $i$ alone. So the study of ir-rotational, pseudo-steady and isentropic fluid flow can proceed along (3.16).

We point out for the case $\gamma=1$ that the dependent variable $i=\ln \rho$, instead of $i=c^{2} /(\gamma-1)$, is used [12]. Then we can obtain a similar equation for $i$,

$$
\left(1-i_{u}^{2}\right) i_{v v}+2 i_{u} i_{v} i_{u v}+\left(1-i_{v}^{2}\right) i_{u u}=i_{u}^{2}+i_{v}^{2}-2 .
$$

We will establish in the pseudo-steady case that the transform is not degenerate, i.e.,

$$
J_{T}(u, v ; \xi, \eta)=\frac{\partial(u, v)}{\partial(\xi, \eta)}=u \xi v_{\eta}-u_{\eta} v \xi \neq 0
$$

in regions of non-simple waves, to be detailed later. In the direction from $(u, v)$ plane to the $(\xi, \eta)$ plane, it is more direct to compute

$$
J_{T}^{-1}(u, v ; \xi, \eta)=\xi_{u} \eta_{v}-\xi_{v} \eta_{u} \neq 0
$$

Noting that (2.3) and (3.14) are all two by two systems, we use Proposition 3.1 to assert that the characteristics of (2.3) are mapped into the characteristics of (3.14) by the hodograph transformation (3.11). Moreover, the eigenvalues of (2.3) are

$$
\Lambda_{ \pm}=\frac{(u-\xi)(v-\eta) \pm c \sqrt{(u-\xi)^{2}+(v-\eta)^{2}-c^{2}}}{(u-\xi)^{2}-c^{2}}
$$

while the eigenvalues of (3.14) are

$$
\lambda_{ \pm}=\frac{i_{u} i_{v} \pm c \sqrt{\left(i_{u}^{2}+i_{v}^{2}-c^{2}\right)}}{c^{2}-i_{v}^{2}} .
$$


By using (3.13), it is easy to see that

$$
\lambda_{ \pm}=-\frac{1}{\Lambda_{\mp}}
$$

Furthermore, there is a correspondence between $\Lambda_{ \pm}$and $\lambda_{ \pm}$. Indeed, let $\eta=\eta(\xi)$ be a characteristic curve in the $(\xi, \eta)$ plane with $\frac{d \eta}{d \xi}=\Lambda_{+}$and be mapped onto a curve $v=v(u)$. Then, using (3.15) and (3.22), we have

$$
\Lambda=\frac{d \eta}{d \xi}=\frac{\eta_{u}+\eta_{v} \frac{d v}{d u}}{\xi_{u}+\xi_{v} \frac{d v}{d u}}
$$

i.e.,

$$
\frac{d v}{d u}=-\frac{\xi_{u} \Lambda_{+}-\eta_{u}}{\xi_{v} \Lambda_{+}-\eta_{v}}=-\frac{\left(1+i_{u u}\right) \Lambda_{+}-i_{u v}}{i_{u v} \Lambda_{+}-\left(1+i_{v v}\right)}=-\frac{i_{u u}+1+\lambda_{-} i_{u v}}{i_{u v}+\lambda_{-}\left(i_{v v}+1\right)} .
$$

We rewrite (3.16) as

$$
i_{u u}+1+\left(\lambda_{-}+\lambda_{+}\right) i_{u v}+\lambda_{-} \lambda_{+}\left(i_{v v}+1\right)=0
$$

Then we conclude

$$
\frac{d v}{d u}=\lambda_{+}
$$

Similarly we obtain the correspondence between $\Lambda_{-}$and $\lambda_{-}$.

\subsection{Steady Euler}

The steady isentropic and irrotational Euler system of (1.1) has the form

$$
\left\{\begin{array}{l}
\left(c^{2}-u^{2}\right) u_{x}-u v\left(u_{y}+v_{x}\right)+\left(c^{2}-v^{2}\right) v_{y}=0 \\
u_{y}-v_{x}=0
\end{array}\right.
$$

where $c$ is the sound speed, given by Bernoulli's law

$$
\frac{u^{2}+v^{2}}{2}+\frac{c^{2}}{\gamma-1}=\frac{k_{0}}{2}
$$

where $k_{0}$ is a constant. See [5]. Using the hodograph transform from $(x, y)$ to $(u, v)$, we obtain a linear system,

$$
\left\{\begin{array}{l}
\left(2 \kappa i-u^{2}\right) y_{v}+u v\left(x_{v}+y_{u}\right)+\left(2 \kappa i-v^{2}\right) x_{u}=0 \\
x_{v}-y_{u}=0
\end{array}\right.
$$

The hodograph transform is valid in the region of non-simple waves. With the same set of steps in deriving (3.13), we obtain

$$
-u=i_{u}, \quad-v=i_{v}
$$

This can also be obtained formally from (3.13) by regarding the steady flow as the limit of unsteady flow (1.1) in $t \rightarrow \infty$. Comparing (3.30) with (3.13), we see that it is much more difficult to convert the hodograph plane of the steady case back into the physical plane than the pseudo-steady case. However, system (3.29) has more advantage over (3.14) of the pseudosteady case because $i$ is expressed in an explicit form by Bernoulli's law (3.28). 


\subsection{Similarity to one-dimensional problems}

The current approach parallels the procedure that is used to find centered rarefaction waves to genuinely nonlinear strictly hyperbolic systems of conservation laws in one space dimension. Recall for a one-dimensional system $u_{t}+f(u)_{x}=0$ of $n$ equations, a centered rarefaction wave takes the form $\xi=\lambda_{k}(u)$ for a $k \in(1, n)$ and the state variable $u$ satisfies the system of ordinary differential equations $\left(f^{\prime}(u)-\lambda_{k}(u) I\right) u_{\xi}=0$, whose solutions are rarefaction wave curves in the phase space. The development (or inversion) of the phase space solutions onto the $\xi$-axis requires the monotonicity of $\lambda_{k}(u)$ along the vector field of the $k$-th right eigenvector $r_{k}$; i.e., the genuine nonlinearity. For the self-similar 2-D Euler system, we have a pair $\xi=u+i_{u}, \eta=$ $v+i_{v}$ from (3.13) in place of $\xi=\lambda_{k}(u)$; and the second-order partial differential equation (3.16) in place of the ordinary differential system. For inversion to the physical space, we show that the Jacobian $J_{T}^{-1}$ of (3.19) does not vanish.

\section{Simple waves}

\subsection{Concept of simple waves}

Now we recall some facts about simple waves. Simple waves were systematically studied, e.g. in [9], for hyperbolic systems in two independent variables,

$$
u_{t}+A(u) u_{x}=0,
$$

where $u=\left(u_{1}, \cdots, u_{n}\right)^{\top}$, the $n \times n$ matrix $A(u)$ has real and distinct eigenvalues $\lambda_{1}<\cdots<\lambda_{n}$ for all $u$ under consideration. They are defined as a special family of solutions of the form

$$
u=U(\phi(x, t)) .
$$

The function $\phi=\phi(x, t)$ is scalar. Substituting (4.2) into (4.1) yields

$$
U^{\prime}(\phi) \phi_{t}+A(U(\phi)) U^{\prime}(\phi) \phi_{x}=0,
$$

which implies that $-\phi_{t} / \phi_{x}$ is an eigenvalue of $A(U(\phi))$ and $U^{\prime}(\phi)$ is the associated eigenvector. This concludes that in the $(x, t)$ plane a simple wave is associated with a kind of characteristic field, say, $\lambda_{k}$, and spans a domain in which characteristics of the $k$-kind are straight along which the solution is constant.

The property of simple waves can be analyzed by using Riemann invariants. A Riemann invariant is a scalar function $w=w(x, t)$ satisfying the following condition,

$$
r_{k} \cdot \operatorname{grad} w=0,
$$

for all values of $u$, where $r_{k}$ is the $k$-th right eigenvector of $A$. Using the Riemann invariants, it can be shown that $a$ state in a domain adjacent to a domain of constant state is always a simple wave. 
In general, system (4.1) is not endowed with a full coordinate system of Riemann invariants such that it is transformed into a diagonal form [6]. Note that in (4.1) the coefficient matrix $A$ depends on $u$ only. Once $A$ depends on $x$ and $t$ as well as $u$, the treatment in [9] and [6] breaks down. For example, we are unable to use the same techniques to show that it is a simple wave to be adjacent to a constant state.

\subsection{Simple waves for pseudo-steady Euler equations}

We introduce in a traditional manner a simple wave for (2.3) as a solution $(u, v)=(u, v)(\xi, \eta)$ that is constant along the level set $l: l(\xi, \eta)=C$ for some function $l(\xi, \eta)$, where $C$ is constant. That is, this solution has the form,

$$
(u, v)(\xi, \eta)=(F, G)(l(\xi, \eta))
$$

Inserting this into (2.3) gives

$$
\left(\begin{array}{cc}
\left(2 \kappa i-U^{2}\right) l_{\xi}-U V l_{\eta}, & -U V l_{\xi}+\left(2 \kappa i-V^{2}\right) l_{\eta} \\
l_{\eta} & -l_{\xi}
\end{array}\right)\left(\begin{array}{c}
F^{\prime} \\
G^{\prime}
\end{array}\right)=0 .
$$

Here we use $U:=u-\xi, V:=v-\eta$ for short. It turns out that $\left(F^{\prime}, G^{\prime}\right)=(0,0)$ or there exists a singular solution for which the coefficient matrix becomes singular. The former just gives a trivial constant solution. But for the latter, $l(\xi, \eta)$ satisfies

$$
\left(2 \kappa i-U^{2}\right) l_{\xi}^{2}-2 U V l_{\xi} l_{\eta}+\left(2 \kappa i-V^{2}\right) l_{\eta}^{2}=0
$$

i.e.,

$$
-\frac{l_{\xi}}{l_{\eta}}=\frac{U V \pm\left\{2 \kappa i\left(U^{2}+V^{2}-2 \kappa i\right)\right\}^{1 / 2}}{U^{2}-2 \kappa i}=: \Lambda_{ \pm},
$$

which implies that the level curves $l(\xi, \eta)=C$ are characteristic lines, and

$$
F^{\prime}+\Lambda_{ \pm} G^{\prime}=0
$$

holds along each characteristic line $l(\xi, \eta)=C$ locally at least.

In a recent paper by Li, Zhang, Zheng [15], the pseudo-steady full Euler is shown to have a characteristic decomposition. Let us quote several identities from that paper. First, the flow will be isentropic and ir-rotational adjacent to a constant state. Then the pseudo-characteristics are defined as

$$
\frac{d \eta}{d \xi}=\frac{U V \pm c \sqrt{U^{2}+V^{2}-c^{2}}}{U^{2}-c^{2}} \equiv \Lambda_{ \pm}
$$

Here $c$ is the speed of sound $c^{2}=\gamma p / \rho$. Regarding $\Lambda_{ \pm}$as simple straight functions of the three independent variables $\left(U, V, c^{2}\right)$, we have

$$
\partial_{U} \Lambda=\Lambda(U \Lambda-V) / \Theta, \partial_{V} \Lambda=(V-U \Lambda) / \Theta, \partial_{c^{2}} \Lambda=-\left(1+\Lambda^{2}\right) /(2 \Theta)
$$


where $\Theta:=\Lambda\left(c^{2}-U^{2}\right)+U V$. Then we further obtain

$$
\begin{gathered}
\partial^{ \pm} u+\Lambda_{\mp} \partial^{ \pm} v=0, \\
\partial^{ \pm} c^{2}=-2 \kappa\left(U \partial^{ \pm} u+V \partial^{ \pm} v\right), \\
\partial^{ \pm} \Lambda_{ \pm}=\left[\partial_{U} \Lambda_{ \pm}-\Lambda_{\mp}^{-1} \partial_{V} \Lambda_{ \pm}-2 \kappa\left(U-V / \Lambda_{\mp}\right) \partial_{c^{2}} \Lambda_{ \pm}\right] \partial^{ \pm} u,
\end{gathered}
$$

where $\partial^{ \pm}=\partial_{\xi}+\Lambda_{ \pm} \partial_{\eta}$. We keep $\partial_{ \pm}$for later use in the hodograph plane. Thus, if one of the quantities $\left(u, v, c^{2}\right)$ is a constant along $\Lambda_{-}$, so are the remaining two and $\Lambda_{-}$. The same is true for the plus family $\Lambda_{+}$. Hence we have

Proposition 4.1 (Section 4, [15]). For the irrotational and isentropic pseudo-steady flow (2.1) or (2.3), we have the following characteristic decomposition

$$
\partial^{+} \partial^{-} u=h \partial^{-} u, \quad \partial^{-} \partial^{+} u=g \partial^{+} u
$$

where $h=h(u, v, c)$ and $g=g(u, v, c)$ are some functions. Similar decompositions hold for $v, c^{2}$ and $\Lambda_{ \pm}$. We further conclude that simple waves are waves such that one family of characteristic curves are straight along which the physical quantities $\left(u, v, c^{2}\right)$ are constant.

\section{Convertibility}

We are now ready to discuss the non-degeneracy of hodograph transformation (3.11).

Theorem 5.1 (Sufficient and Necessary Condition). Let the ir-rotational, isentropic and pseudo-steady fluid flow (2.1) be smooth at a point $(\xi, \eta)=\left(\xi_{0}, \eta_{0}\right)$. Then the Jacobian $J_{T}(u, v ; \xi, \eta)$ of the hodograph transformation (3.11) vanishes in a neighborhood of the point if the flow is a simple wave in the neighborhood. Conversely, if the Jacobian $J_{T}(u, v ; \xi, \eta)$ vanishes in a neighborhood of the point, then the flow is a simple wave in the neighborhood.

Proof. Assume first that $c^{2}-V^{2} \neq 0$ at $(\xi, \eta)=\left(\xi_{0}, \eta_{0}\right)$. We compute

$$
\begin{aligned}
& J_{T}(u, v ; \xi, \eta)=u \xi v_{\eta}-u_{\eta} v_{\xi} \\
& =-\frac{1}{c^{2}-V^{2}} \cdot\left[\left(\left(c^{2}-U^{2}\right) u_{\xi}-2 U V u_{\eta}\right) u_{\xi}\right]-u_{\eta}^{2} \\
& =-\frac{1}{c^{2}-V^{2}} \cdot\left[\left(c^{2}-U^{2}\right) u_{\xi}^{2}-2 U V u_{\xi} u_{\eta}+\left(c^{2}-V^{2}\right) u_{\eta}^{2}\right] .
\end{aligned}
$$

Therefore, the degeneracy of the transformation implies

$$
\left(c^{2}-U^{2}\right) u_{\xi}^{2}-2 U V u \xi u_{\eta}+\left(c^{2}-V^{2}\right) u_{\eta}^{2}=0 .
$$

It follows that

$$
-\frac{u \xi}{u_{\eta}}=\Lambda_{ \pm}
$$


That is,

$$
u_{\xi}+\Lambda_{+} u_{\eta}=0, \text { or } u_{\xi}+\Lambda_{-} u_{\eta}=0,
$$

at $\left(\xi_{0}, \eta_{0}\right)$. For the former, we deduce that $\partial^{+} u=0$ along the whole $\Lambda_{-}$-characteristic line through $\left(\xi_{0}, \eta_{0}\right)$ in view of (4.15) in Proposition 4.1, and so do $\partial^{+} v$ and $\partial^{+} c$. Therefore, conclude that the wave is a simple wave associated with $\Lambda_{+}$.

Conversely, if a point $(\xi, \eta)=\left(\xi_{0}, \eta_{0}\right)$ is in the region of a simple wave, then equation (5.4) hold either for the plus or minus families. From there we go up the derivation to find that the Jacobian vanishes in the same neighborhood.

The case that $c^{2}-(v-\eta)^{2}=0$ is a special planar simple wave. Therefore the conclusion follows naturally.

We comment that the Jacobian $J_{T}(u, v ; \xi, \eta)$ can be factorized as

$$
J_{T}(u, v ; \xi, \eta)=-\frac{1}{\Lambda_{-} \Lambda_{+}} \partial^{+} u \cdot \partial^{-} u=-\partial^{+} v \cdot \partial^{-} v
$$

\section{Phase space system of equations}

In this section we use the inclination angles of characteristics as useful variables to rewrite (3.16) in the hodograph plane. We proceed as follows. We first transform the second order equation (3.16) into a first-order system of equations as in [13]. Introduce

$$
X=i_{u}, \quad Y=i_{v} .
$$

Then we deduce a $3 \times 3$ system of first order equations,

$$
\begin{gathered}
{\left[\begin{array}{ccc}
2 \kappa i-Y^{2} & X Y & 0 \\
0 & 1 & 0 \\
0 & 0 & 1
\end{array}\right]\left[\begin{array}{c}
X \\
Y \\
i
\end{array}\right]_{u}+\left[\begin{array}{ccc}
X Y & 2 \kappa i-X^{2} & 0 \\
-1 & 0 & 0 \\
0 & 0 & 0
\end{array}\right]\left[\begin{array}{c}
X \\
Y \\
i
\end{array}\right]_{v}} \\
=\left[\begin{array}{c}
X^{2}+Y^{2}-4 \kappa i \\
0 \\
X
\end{array}\right]
\end{gathered}
$$

This system is equivalent to (3.16) for $C^{1}$ solutions if the given datum for $Y$ is compatible with the datum for $i_{v}$. This system has three eigenvalues

$$
\begin{aligned}
& \lambda_{0}=0 \\
& \frac{d v}{d u}=\lambda_{ \pm}=\frac{X Y \pm \sqrt{2 \kappa i\left(X^{2}+Y^{2}-2 \kappa i\right)}}{2 \kappa i-Y^{2}}=\frac{2 \kappa i-X^{2}}{X Y \mp \sqrt{2 \kappa i\left(X^{2}+Y^{2}-2 \kappa i\right)}}
\end{aligned}
$$


from which we deduce that (6.2) is hyperbolic if $X^{2}+Y^{2}-2 \kappa i>0$ provided that $i>0$ and $2 \kappa i-Y^{2} \neq 0$ (or $2 \kappa i-X^{2} \neq 0$ ). If $2 \kappa i-Y^{2}=0$ or $2 \kappa i-X^{2}=0$, we have planar rarefaction waves in the neighborhood. The three associated left eigenvectors with (6.3) are

$$
l_{0}=(0,0,1), \quad l_{\mp}=\left(\mp 1, \sqrt{2 \kappa i\left(X^{2}+Y^{2}-2 \kappa i\right)}, 0\right) .
$$

We multiply (6.2) by the left eigen matrix $M=\left(l_{+}, l_{-}, l_{0}\right)^{\top}$ (here and onward the superscript $\top$ means transpose) from the left-hand side to obtain

$$
\left\{\begin{array}{l}
X_{u}+\lambda_{-} Y_{u}+\lambda_{+}\left(X_{v}+\lambda_{-} Y_{v}\right)=\frac{X^{2}+Y^{2}-4 \kappa i}{2 \kappa i-Y^{2}} \\
X_{u}+\lambda_{+} Y_{u}+\lambda_{-}\left(X_{v}+\lambda_{+} Y_{v}\right)=\frac{X^{2}+Y^{2}-4 \kappa i}{2 \kappa i-Y^{2}} \\
i_{u}=X
\end{array}\right.
$$

Introduce the inclination angles $\alpha, \beta(-\pi / 2<\alpha, \beta<\pi / 2)$ of $\Lambda_{+}$and $\Lambda_{-}$-characteristics by

$$
\tan \alpha=\Lambda_{+}, \quad \tan \beta=\Lambda_{-} .
$$

Note that, see (3.22),

$$
\Lambda_{+}=-\frac{1}{\lambda_{-}}, \quad \Lambda_{-}=-\frac{1}{\lambda_{+}}
$$

and denote

$$
A:=\tan (\alpha / 2), \quad B:=\tan (\beta / 2) .
$$

This explains the Riemann invariant introduced in [13]. Then we find that $X, Y$ are related with $A, B$ through the following identities,

$$
\begin{aligned}
& A=\frac{X-\sqrt{X^{2}+Y^{2}-c^{2}}}{c-Y}, \\
& B=-\frac{X-\sqrt{X^{2}+Y^{2}-c^{2}}}{c+Y}, \quad\left(c^{2}=2 \kappa i\right),
\end{aligned}
$$

or

$$
X=\frac{c(1-A B)}{A-B}, \quad Y=\frac{c(A+B)}{A-B} .
$$

In terms of $\alpha, \beta$, we have

$$
X=c \frac{\cos \left(\frac{\alpha+\beta}{2}\right)}{\sin \left(\frac{\alpha-\beta}{2}\right)}, \quad Y=c \frac{\sin \left(\frac{\alpha+\beta}{2}\right)}{\sin \left(\frac{\alpha-\beta}{2}\right)} .
$$


We observe that the variables $\alpha, \beta$ are Riemann invariants for (6.5). In fact, we can write (6.5) as

$$
\begin{aligned}
& \partial_{+} \alpha=\frac{1+\kappa}{2 c} \cdot \frac{\sin (\alpha-\beta)}{\sin \beta} \cdot\left[m-\tan ^{2}\left(\frac{\alpha-\beta}{2}\right)\right] \\
& \partial_{-} \beta=\frac{1+\kappa}{2 c} \cdot \frac{\sin (\alpha-\beta)}{\sin \beta} \cdot\left[m-\tan ^{2}\left(\frac{\alpha-\beta}{2}\right)\right] \\
& \partial_{0} c=\kappa \frac{\cos \left(\frac{\alpha+\beta}{2}\right)}{\sin \left(\frac{\alpha-\beta}{2}\right)}
\end{aligned}
$$

where we use the notations of directional derivatives,

$$
\partial_{+}=\frac{\partial}{\partial u}+\lambda_{+} \frac{\partial}{\partial v}, \quad \partial_{-}=\frac{\partial}{\partial u}+\lambda_{-} \frac{\partial}{\partial v}, \quad \partial_{0}=\frac{\partial}{\partial u}
$$

and keep the letter $m$ for

$$
m=\frac{1-\kappa}{1+\kappa}=\frac{3-\gamma}{1+\gamma}
$$

We further introduce the normalized directional derivatives along characteristics,

$$
\bar{\partial}_{+}=(\sin \beta,-\cos \beta) \cdot\left(\partial_{u}, \partial_{v}\right), \quad \bar{\partial}_{-}=(\sin \alpha,-\cos \alpha) \cdot\left(\partial_{u}, \partial_{v}\right)
$$

They are coordinate-free. Using them, we write (6.12) as,

$$
\begin{aligned}
\bar{\partial}_{+} \alpha & =\frac{1+\kappa}{2 c} \cdot \sin (\alpha-\beta) \cdot\left[m-\tan ^{2}\left(\frac{\alpha-\beta}{2}\right)\right]=: G(\alpha, \beta, c), \\
\bar{\partial}_{-} \beta & =\frac{1+\kappa}{2 c} \cdot \sin (\alpha-\beta) \cdot\left[m-\tan ^{2}\left(\frac{\alpha-\beta}{2}\right)\right] \equiv G(\alpha, \beta, c), \\
\partial_{0} c & =\kappa \frac{\cos \left(\frac{\alpha+\beta}{2}\right)}{\sin \left(\frac{\alpha-\beta}{2}\right)} .
\end{aligned}
$$

In particular, we note that

$$
\bar{\partial}_{+} c=-\kappa, \quad \bar{\partial}_{-} c=\kappa .
$$

This highlights that the first two equations of (6.16) are entirely decoupled from the third $c$ equation. In addition, each of the first two equations of (6.16) is actually a decomposition of the second-order equation (3.16) for $c$.

Note from (6.6) and (6.7) that $\lambda_{+}=-\cot \beta$ and $\lambda_{-}=-\cot \alpha$. The system (6.12) is linearly degenerate in the sense of Lax [9]. For the particular case that $(\alpha-\beta) / 2=(1-\kappa) /(1+\kappa)$ for $1<\gamma<3$, the first two equations become homogeneous equations

$$
\begin{aligned}
& \alpha_{u}+\lambda_{+} \alpha_{v}=0, \\
& \beta_{u}+\lambda_{-} \beta_{v}=0,
\end{aligned}
$$


which always have a unique global continuous solution provided that the corresponding initial and/or boundary data have a uniform bound in $C^{1}$ norm (cf. [17]). In fact, the explicit solutions of Suchkov [24] in the expansion problem of a wedge of gas into a vacuum is such a case, see Remark 7.1 in Section 7.

The mapping $(X, Y) \rightarrow(\alpha, \beta)$ is bijective as long as system (6.5) is hyperbolic.

We summarizes the above as follows, which is similar to [13]:

Theorem 6.1. The two-dimensional pseudo-steady, irrotational, isentropic flow (3.16) can be transformed into a linearly degenerate system of first order partial differential equations (6.12) or (6.16) provided that the transform $(X, Y) \rightarrow(\alpha, \beta)$ is invertible, i.e., system (6.5) is hyperbolic.

Regarding $\bar{\partial}_{-} \alpha$ and $\bar{\partial}_{+} \beta$, we have second-order equations although we are unable to obtain explicit expressions for them like (6.16). By direct computations, we obtain

Lemma 6.1 (Commutator relation of $\left.\partial_{ \pm}\right)$. For any quantity $I=I(u, v)$, there holds

$$
\partial_{-} \partial_{+} I-\partial_{+} \partial_{-} I=\frac{\partial_{-} \lambda_{+}-\partial_{+} \lambda_{-}}{\lambda_{-}-\lambda_{+}}\left(\partial_{-} I-\partial_{+} I\right)
$$

Lemma 6.2 (Commutator relation of $\left.\bar{\partial}_{ \pm}\right)$. For any quantity $I=I(u, v)$, there holds,

$$
\bar{\partial}_{-} \bar{\partial}_{+} I-\bar{\partial}_{+} \bar{\partial}_{-} I=\frac{1-\cos (\alpha-\beta)}{\sin (\alpha-\beta)}\left(\bar{\partial}_{-} I+\bar{\partial}_{+} I\right) \bar{\partial}_{+} \alpha
$$

where $\bar{\partial}_{+} \alpha$ is given in (6.16). Noting $\bar{\partial}_{+} \alpha=\bar{\partial}_{-} \beta$ in (6.16), we can also use $\bar{\partial}_{-} \beta$ in (6.20).

Using these commutator relations, we easily derive:

Theorem 6.2. Assume that the solution of (6.16) $(\alpha, \beta) \in C^{2}$. Then we have

$$
\begin{aligned}
\bar{\partial}_{+} \bar{\partial}_{-} \alpha+W \bar{\partial}_{-} \alpha & =Q(\alpha, \beta, c), \\
-\bar{\partial}_{-} \bar{\partial}_{+} \beta+W \bar{\partial}_{+} \beta & =Q(\alpha, \beta, c),
\end{aligned}
$$

where $W(\alpha, \beta, c)$ and $Q(\alpha, \beta, c)$ are

$$
\begin{aligned}
W(\alpha, \beta, c) & =\frac{1+\kappa}{2 c}\left[\left(m-\tan ^{2} \omega\right)\left(3 \tan ^{2} \omega-1\right) \cos ^{2} \omega+2 \tan ^{2} \omega\right], \\
Q(\alpha, \beta, c) & =\frac{(1+\kappa)^{2}}{4 c^{2}} \sin (2 \omega)\left(m-\tan ^{2} \omega\right)\left(3 \tan ^{2} \omega-1\right), \\
\omega & =\frac{\alpha-\beta}{2} .
\end{aligned}
$$


Proof. The proof is simple. Recall from (6.17) that

$$
\bar{\partial}_{+} c=-\kappa, \quad \bar{\partial}_{-} c=\kappa .
$$

Then we apply the commutator relation to obtain (setting $I=\alpha$ in (6.20))

$$
\bar{\partial}_{+} \bar{\partial}_{-} \alpha=\bar{\partial}_{-} \bar{\partial}_{+} \alpha+\frac{1-\cos (\alpha-\beta)}{\sin (\beta-\alpha)}\left(\bar{\partial}_{-} \alpha+\bar{\partial}_{+} \alpha\right) \bar{\partial}_{-} \beta
$$

Using the expressions of $\bar{\partial}_{+} \alpha$ and $\bar{\partial}_{-} \beta$ in (6.16), we compute directly to yield the result in (6.21) and the proof of Theorem 6.2 is complete.

\section{The gas expansion problem}

We now use the hodograph transformation and the decomposition of the previous section to study the expansion of a wedge of gas into vacuum. The problem was studied earlier in $[24,19,11,13]$, and especially by $\mathrm{Li}$ in $[12,13]$, but the solution of $\mathrm{Li}$ is in the hodograph plane for $1 \leq \gamma<3$, and the behavior of the vacuum boundary was left open. We continue the effort of $\mathrm{Li}$ and prove that the solution in the hodograph plane can be transformed back to the physical self-similar plane for all $\gamma>1$ and the vacuum boundary is a Lipschitz continuous curve which is monotone in the upper and lower parts of the wedge respectively. We also determine explicitly the relative location of the vacuum boundary with respect to the vertical position of the explicit solution of Suchkov [24]. Moreover, we can draw a clear picture of the distribution of characteristics. For notational simplicity in this section, we use $\bar{m}, m_{0}$, defined by

$$
\tan ^{2} \bar{m}=m, \quad m_{0}=1 / \sqrt{m},
$$

for $1<\gamma<3$; and $\bar{m} \equiv 0$ for $\gamma \geq 3$.

\subsection{The planar rarefaction waves.}

First we prepare our planar rarefaction waves. Assume that the initial data for (1.1) is

$$
(\rho, u, v)(x, y, 0)= \begin{cases}\left(\rho_{1}, 0,0\right), & \text { for } n_{1} x+n_{2} y>0 \\ \text { vacuum, }, & \text { for } n_{1} x+n_{2} y<0\end{cases}
$$

where $n_{1}^{2}+n_{2}^{2}=1$, and $\rho_{1}$ is a constant. The solution of (1.1) and (7.2) takes the form, see [14],

$$
(\rho, u, v)(x, y, t)= \begin{cases}\left(\rho_{1}, 0,0\right), & \zeta>1, \\ (\rho, u, v)(\zeta), & -1 / \kappa \leq \zeta \leq 1 \\ \text { vacuum }, & \zeta<-1 / \kappa\end{cases}
$$


where $\zeta=n_{1} \xi+n_{2} \eta,(\xi, \eta)=(x / t, y / t)$, and the solution $(c, u, v)$ has been normalized so that $c_{1}=1$. The rarefaction wave solution $(\rho, u, v)(\zeta)$ satisfies

$$
\zeta=n_{1} u+n_{2} v+c, \quad \frac{n_{1}}{\kappa} c-u=\frac{n_{1}}{\kappa}, \quad \frac{n_{2}}{\kappa} c-v=\frac{n_{2}}{\kappa} .
$$

Note that this rarefaction wave corresponds to a segment in the hodograph plane, $n_{2} u-n_{1} v=$ $0,-n_{1} / \kappa \leq u \leq 0$.

In particular, when we consider the rarefaction wave propagates in the $x$-direction, i.e., $\left(n_{1}, n_{2}\right)=(1,0)$, this wave can be expressed as

$$
x / t=u+c, \quad c=\kappa u+1, \quad v \equiv 0, \quad-1 / \kappa \leq u \leq 0 .
$$

That is, in the hodograph $(u, v)$ plane, this rarefaction wave is mapped onto a segment $v \equiv 0$, $-1 / \kappa \leq u \leq 0$, on which we have

$$
i=\frac{1}{2 \kappa}(\kappa u+1)^{2}, \quad i_{u}=\kappa u+1, \quad i_{u u}=\kappa .
$$

When we consider the expansion problem of a wedge of gas in the next subsection, we need to know not only the derivatives of $i$ with respect to $u$ in (7.6), but also the derivatives with respect to $v$, on the segment $v \equiv 0,-1 / \kappa \leq u \leq 0$. For this purpose, we insert (7.6) into (3.16) to obtain

$$
\left(i_{v}^{2}\right)_{u}-\frac{\kappa+1}{\kappa u+1} i_{v}^{2}=-(\kappa+1)(\kappa u+1) .
$$

Solving this equation in terms of $i_{v}^{2}$ yields,

$$
i_{v}^{2}= \begin{cases}(\kappa u+1)^{2}\left[\frac{1}{m}+\left(C^{2}-\frac{1}{m}\right)(\kappa u+1)^{\frac{1-\kappa}{\kappa}}\right], & \text { for } \gamma \neq 3 \\ (1+u)^{2}\left[C^{2}-2 \ln (1+u)\right], & \text { for } \gamma=3\end{cases}
$$

where $C$ is an integral constant. This was obtained in [11].

\subsection{A wedge of gas}

We place the wedge symmetrically with respect to the $x$-axis and the sharp corner at the origin, as in Figure 7.1(a). This problem is then formulated mathematically as seeking the solution of (1.1) with the initial data,

$$
(i, u, v)(t=0, x, y)= \begin{cases}\left(i_{0}, u_{0}, v_{0}\right), & -\theta<\delta<\theta \\ (0, \bar{u}, \bar{v}), & \text { otherwise }\end{cases}
$$

where $i_{0}>0, u_{0}$ and $v_{0}$ are constant, $(\bar{u}, \bar{v})$ is the velocity of the wave front, not being specified in the state of vacuum, $\delta=\arctan y / x$ is the polar angle, and $\theta$ is the half-angle of the wedge 


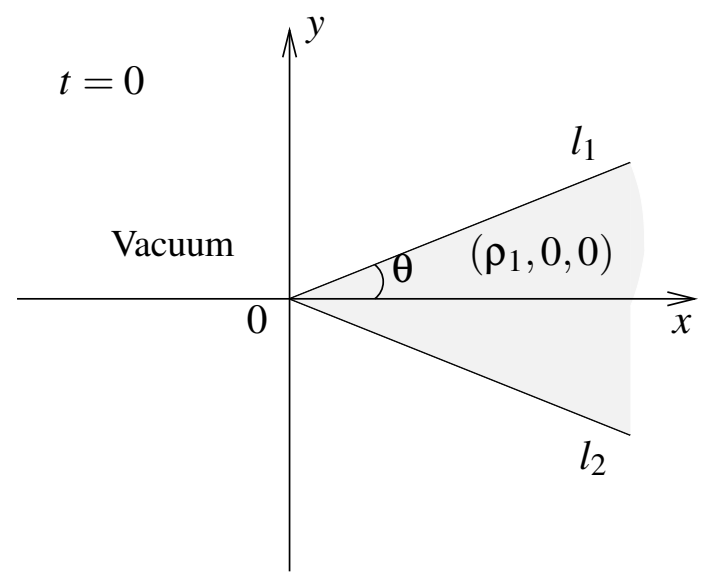

(a) Initial Data

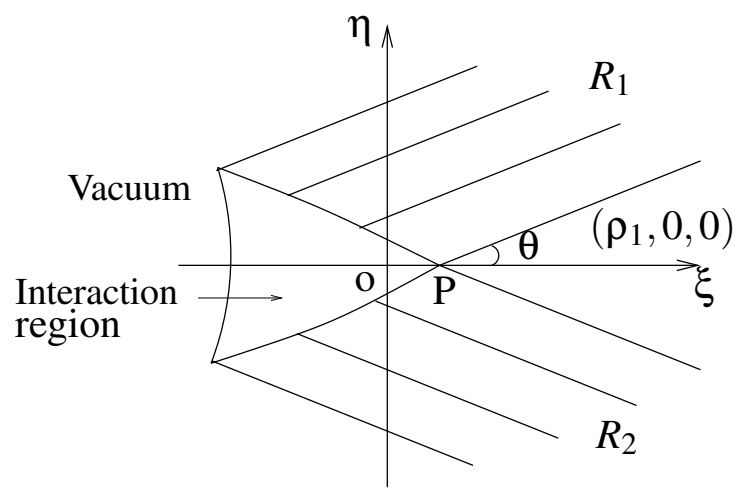

(b) Interaction of rarefaction waves

Figure 7.1: The expansion of a wedge of gas

restricted between 0 and $\pi / 2$. This can be considered as a two-dimensional Riemann problem for (1.1) with two pieces of initial data (7.9). As we will see below, this problem is actually the interaction of two whole planar rarefaction waves. See Figure 7.1(b). We note that the solution we construct is valid for any "portions" of (7.9) as the solutions are hyperbolic.

The gas away from the sharp corner expands into the vacuum as planar rarefaction waves $R_{1}$ and $R_{2}$ of the form $(i, u, v)(t, x, y)=(i, u, v)(\zeta)\left(\zeta=\left(n_{1} x+n_{2} y\right) / t\right)$ where $\left(n_{1}, n_{2}\right)$ is the propagation direction of waves. We assume that initially the gas is at rest, i.e., $\left(u_{0}, v_{0}\right)=$ $(0,0)$. Otherwise, we replace $(u, v)$ by $\left(u-u_{0}, v-v_{0}\right)$ and $(\xi, \eta)$ by $\left(\xi-u_{0}, \eta-v_{0}\right)$ in the following computations (see also (2.1)). We further assume that the initial sound speed is unit since the transformation $(u, v, c, \xi, \eta) \rightarrow c_{0}(u, v, c, \xi, \eta)$ with $c_{0}>0$ can make all variables dimensionless. Then the rarefaction waves $R_{1}, R_{2}$ emitting from the initial discontinuities $l_{1}, l_{2}$ are expressed in (7.4) with $\left(n_{1}, n_{2}\right)=(\sin \theta,-\cos \theta)$ and $\left(n_{1}, n_{2}\right)=(\sin \theta, \cos \theta)$, respectively. These two waves begin to interact at $P=(1 / \sin \theta, 0)$ in the $(\xi, \eta)$ plane due to the presence of the sharp corner and a wave interaction region, called the wave interaction region $\mathcal{D}$, is formed to separate from the planar rarefaction waves by $k_{1}, k_{2}$,

$$
\begin{aligned}
& k_{1}:\left(1-\kappa^{2}\right) \xi_{1}^{2}-\left(\kappa \eta_{1}+1\right)^{2}=2^{(1-\kappa) / \kappa} C\left(\kappa \eta_{1}+1\right)^{(\kappa+1) / \kappa}, \quad\left(\xi_{1}>0,-1 \leq \eta_{1} \leq 1 / \kappa\right), \\
& k_{2}:\left(1-\kappa^{2}\right) \xi_{2}^{2}-\left(\kappa \eta_{2}+1\right)^{2}=2^{(1-\kappa) / \kappa} C\left(\kappa \eta_{2}+1\right)^{(\kappa+1) / \kappa}, \quad\left(\xi_{2}>0,-1 / \kappa \leq \eta_{2} \leq 1\right),
\end{aligned}
$$

where $k_{1}$ and $k_{2}$ are two characteristics from $P$, associated with the nonlinear eigenvalues of system $(2.1)$, see $[14,26]$, and the constant $C$ is

$$
\begin{aligned}
C= & (\gamma+1)\left[\frac{1}{\sqrt{\gamma}(\gamma+1)}\right]^{(\gamma+1) /(\gamma-1)} \\
& \cdot\left[(3-\gamma)(\gamma)^{-(\gamma+1) /(2(\gamma-1))}+(\gamma+1)(\gamma)^{(\gamma-3) /(2(\gamma-1))}\right],
\end{aligned}
$$


and

$$
\left\{\begin{array} { l } 
{ \xi _ { 1 } = \xi \operatorname { c o s } \theta + \eta \operatorname { s i n } \theta , } \\
{ \eta _ { 1 } = - \xi \operatorname { s i n } \theta + \eta \operatorname { c o s } \theta , }
\end{array} \quad \left\{\begin{array}{l}
\xi_{2}=\xi \cos \theta-\eta \sin \theta \\
\eta_{2}=\xi \sin \theta+\eta \cos \theta
\end{array}\right.\right.
$$

So, the wave interaction region $\mathcal{D}$ is bounded by $k_{1}, k_{2}$ and the interface of gas with vacuum, connecting $D$ and $E$, see Figure 7.1(b). The solution outside $\mathcal{D}$ consists of the constant state $\left(i_{0}, u_{0}, v_{0}\right)$, the vacuum, and the planar rarefaction waves $R_{1}$ and $R_{2}$.

Problem A. Find a solution of (2.1) inside the wave interaction region $\mathcal{D}$, subject to the boundary values on $k_{1}$ and $k_{2}$, which are determined continuously from the rarefaction waves $R_{1}$ and $R_{2}$.

This problem is a Goursat-type problem for (2.1) since $k_{1}$ and $k_{2}$ are characteristics. Our strategy to solve this problem is to use the hodograph transform, solve the associated problem in the hodograph plane, and show that the hodograph transformation is invertible.

Note that initial data (7.9) is ir-rotational, we conclude that the flow is always ir-rotational provided that it is continuous. So the irrotationality condition (2.2) holds and all results about the hodograph transformation can be used to treat this problem. Then Problem A can be converted into a problem in the hodograph plane.

For this purpose, we need to map the wave interaction region $\mathcal{D}$ in the $(\xi, \eta)$ plane into a region $\Omega$ in the $(u, v)$ plane. Notice that the mapping of the planar rarefaction waves $R_{1}$ and $R_{2}$ into $(u, v)$ plane are exactly two segments

$$
\begin{array}{ll}
H_{1}: u \cos \theta+v \sin \theta=0, & (-\sin \theta / \kappa \leq u \leq 0) \text { and } \\
H_{2}: u \cos \theta-v \sin \theta=0, & (-\sin \theta / \kappa \leq u \leq 0) .
\end{array}
$$

The boundary values of $c$ on $H_{1}, H_{2}$, are

$$
\left.c\right|_{H_{1}}=1+\kappa v^{\prime}=: c_{0}^{1},\left.\quad c\right|_{H_{2}}=1+\kappa v^{\prime \prime}=: c_{0}^{2},
$$

where $v^{\prime}=u \sin \theta-v \cos \theta$ and $v^{\prime \prime}=u \sin \theta+v \cos \theta$. Obviously,

$$
0 \leq c_{0}^{1}, c_{0}^{2} \leq 1
$$

Thus the wave interaction region $\Omega$ is bounded by $H_{1}, H_{2}$ and the interface of vacuum connecting $D$ and $E$ in the hodograph $(u, v)$-plane, see Figure 7.2. We define $\Omega$ more precisely to contain the boundaries $H_{1}$ and $H_{2}$, but not the vacuum boundary $c=0$.

Boundary conditions. We need to derive the necessary boundary conditions on $H_{1}$ and $\mathrm{H}_{2}$, respectively. This can be done simply by using coordinate transformations for (7.6) and (7.8). Indeed, denote temporarily (cf. (7.8))

$$
\Gamma(u, C):= \begin{cases}{\left[\frac{1}{m}+\left(C^{2}-\frac{1}{m}\right)(\kappa u+1)^{\frac{1-\kappa}{\kappa}}\right]^{\frac{1}{2}},} & \text { for } \gamma \neq 3, \\ {\left[C^{2}-2 \ln (1+u)\right]^{\frac{1}{2}},} & \text { for } \gamma=3,\end{cases}
$$




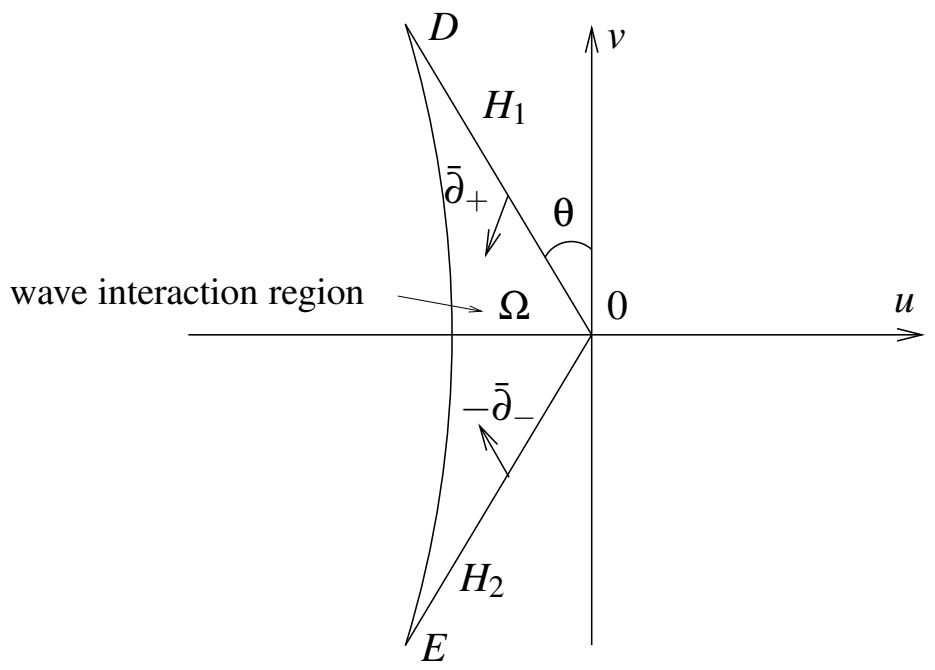

Figure 7.2: Wave interaction region in the hodograph plane

Then we have

$$
\begin{aligned}
& i_{u}=\left(1-\kappa v^{\prime}\right)\left\{\Gamma\left(v^{\prime}, C_{1}\right) \cos \theta+\sin \theta\right\}, \\
& i_{v}=\left(1-\kappa v^{\prime}\right)\left\{\left(\Gamma\left(v^{\prime}, C_{1}\right) \sin \theta-\cos \theta\right\},\right.
\end{aligned}
$$

and

$$
\begin{aligned}
& i_{u}=\left(1+\kappa v^{\prime \prime}\right)\left\{\Gamma\left(v^{\prime \prime}, C_{2}\right) \cos \theta+\sin \theta\right\}, \\
& i_{v}=\left(1+\kappa v^{\prime \prime}\right)\left\{-\Gamma\left(v^{\prime \prime}, C_{2}\right) \sin \theta+\cos \theta\right\},
\end{aligned}
$$

where $C_{1}$ and $C_{2}$ are two constants. Applying the compatibility condition that $i_{u}, i_{v}$ are continuous at $(u, v)=(0,0)$, we obtain

$$
C_{1}=-C_{2}=\cot \theta
$$

Thus we obtain the boundary conditions as in (7.16) and (7.17).

In order to evaluate the boundary values of $\alpha, \beta$, we substitute (7.13), (7.16)-(7.17) into (6.9) to deduce

$$
\begin{aligned}
\left.A\right|_{H_{1}} & =\frac{\sin \theta}{1+\cos \theta}=\tan (\theta / 2), \\
\left.B\right|_{H_{1}} & =-\frac{-\Gamma\left(v^{\prime}, \cot \theta\right) \sin \theta+(1+\cos \theta)}{\Gamma\left(v^{\prime}, \cot \theta\right)(1+\cos \theta)+\sin \theta}=: B_{1} \\
\left.A\right|_{H_{2}} & =\frac{-\Gamma\left(v^{\prime \prime}, \cot \theta\right) \sin \theta+(1+\cos \theta)}{\Gamma\left(v^{\prime \prime}, \cot \theta\right)(1+\cos \theta)+\sin \theta}=: A_{2}, \\
\left.B\right|_{H_{2}} & =-\frac{1+\cos \theta}{\sin \theta}=-\tan (\theta / 2)
\end{aligned}
$$

Thus the boundary values for $\alpha, \beta$ on $H_{1}$ and $H_{2}$ are

$$
\begin{aligned}
& \left.\alpha\right|_{H_{1}}=\theta,\left.\quad \quad \beta\right|_{H_{1}}=2 \arctan \left(-B_{1}\right), \\
& \left.\alpha\right|_{H_{2}}=2 \arctan \left(A_{2}\right),\left.\quad \beta\right|_{H_{2}}=-\theta \text {. }
\end{aligned}
$$


The boundary values of $c$ on $H_{1}$ and $H_{2}$ are given in (7.13). Now Problem A becomes:

Problem B. Find a solution $(\alpha, \beta, c)$ of (6.12) with boundary values (7.20) and (7.13), in the wave interaction region $\Omega$ in the hodograph plane.

In order to solve Problem B, we estimate the boundary values (7.20) and (7.13).

Lemma 7.1 (Boundary data estimate). For the boundary data (7.20) on the boundaries $H_{i}$, $i=1,2$, we have the following estimates:

(i) If $\theta<\bar{m}$, there holds

$$
2 \theta \leq\left.(\alpha-\beta)\right|_{H_{i}} \leq 2 \bar{m}
$$

(ii) If $\theta>\bar{m}$, there holds

$$
2 \bar{m} \leq\left.(\alpha-\beta)\right|_{H_{i}} \leq 2 \theta
$$

Proof. For the first case, i.e., $\theta<\bar{m}$, by noting $0 \leq 1+\kappa v^{\prime}, 1+\kappa v^{\prime \prime} \leq 1$, we estimate to get

$$
\tan (\theta / 2) \leq\left. B\right|_{H_{1}} \leq \frac{-m_{0} \tan (\theta / 2)+1}{m_{0}+\tan (\theta / 2)}=: m_{\theta}
$$

where $m_{0}=1 / \sqrt{m}$. It is easy to check that

$$
\tan \left(\theta / 2+\arctan m_{\theta}\right)=\sqrt{m}=\tan \bar{m} .
$$

Therefore

$$
2 \theta \leq\left.(\alpha-\beta)\right|_{H_{1}} \leq 2 \bar{m}
$$

Similarly we can prove the second inequality on $H_{2}$ in (7.21).

For the second case that $\theta>\bar{m}$, the proof is also similar if $1<\gamma<3$. If $\gamma \geq 3$, it is evident that

$$
-\tan (\theta / 2) \leq\left. A\right|_{H_{2}},\left.B\right|_{H_{1}} \leq \tan (\theta / 2)
$$

Then the proof is complete.

The local existence of solutions at the origin $(u, v)=(0,0)$ follows routinely from the idea [18, Chapter 2] or [25]. We need only to check the compatibility condition to this problem, i.e.,

$$
\frac{1}{\lambda_{+}}\left[l^{0} \cdot \partial_{+} K-\kappa \frac{\cos \left(\frac{\alpha+\beta}{2}\right)}{\sin \left(\frac{\alpha-\beta}{2}\right.}\right]=\frac{1}{\lambda_{-}}\left[l^{0} \cdot \partial_{-} K-\kappa \frac{\cos \left(\frac{\alpha+\beta}{2}\right)}{\sin \left(\frac{\alpha-\beta}{2}\right)}\right]
$$

at $(u, v)=(0,0)$, where $K=(\alpha, \beta, c)^{\top}$ and $l^{0}=(0,0,1)$. That is, we need to check if there holds

$$
\frac{1}{\lambda_{+}}\left[\partial_{+} c-\kappa \frac{\cos \left(\frac{\alpha+\beta}{2}\right)}{\sin \left(\frac{\alpha-\beta}{2}\right.}\right]=\frac{1}{\lambda_{-}}\left[\partial_{-} c-\kappa \frac{\cos \left(\frac{\alpha+\beta}{2}\right)}{\sin \left(\frac{\alpha-\beta}{2}\right)}\right] .
$$


This is obviously true by using (6.17). Hence we have

Lemma 7.2 (Local existence). There is a $\delta>0$ such that the $C^{1}$-solution of (6.12) and (7.13), (7.20) exists uniquely in the region $\bar{\Omega}=\{(u, v) \in \Omega ;-\delta<u<0\}$, where $\delta$ depends only on the $C^{0}$ and $C^{1}$ norms of $\alpha, \beta$ on the boundaries $H_{1}$ and $H_{2}$.

We do not give the proof. For details, see [18, Chapter 2] or [25].

Next we will extend the local solution to the whole region $\Omega$. Therefore some a priori estimates on the $C^{0}$ and $C^{1}$ norms of $\alpha, \beta$ and $i$, are needed. The norm of $i$ comes from the norms of $\alpha$ and $\beta$, see the third equation of (6.12). Therefore we need only the estimate on $\alpha$ and $\beta$. Recall that the derivation of (6.12) is based on the strict hyperbolicity of the flow, $i>0$. These will be achieved when we estimate the $C^{0}$ norms of $\alpha$ and $\beta$, see Subsection 7.3. The main existence theorem is stated as follows. Let $l$ be the interface of the gas with the vacuum.

Theorem 7.1 (Global existence in the hodograph plane). There exists a solution $(\alpha, \beta, i) \in$ $C^{1}$ to the boundary value problem (6.12) with boundary values (7.13) and (7.20)(Problem B) in $\Omega$. The vacuum interface l exists and is Lipschitz continuous.

We prove this theorem by two steps. We estimate the solution itself in Subsection 7.3 and then proceed with estimates on the gradients in Subsection 7.4. The proof of Theorem 7.1 is also given in Subsection 7.4.

After we solve Problem $B$, we show the inversion of hodograph transformation in Subsection 7.5, which establishes the existence of the gas expansion problem, Problem A.

Theorem 7.2 (Global existence in the physical plane). There exists a solution $(c, u, v) \in C^{1}$ of (2.1) for the gas expansion problem (Problem $A$ ) in the wave interaction region $\mathcal{D}$ in the physical plane, the $(\xi, \eta)$-plane.

\subsection{The maximum norm estimate on $(\alpha, \beta, c)$}

We estimate the solution $(\alpha, \beta, c)$ itself, i.e, the $C^{0}$ norm of $\alpha, \beta$ and $c$. We adopt the method of invariant regions [23].

Lemma 7.3. Suppose that there exists a $C^{1}$ solution $(\alpha(u, v), \beta(u, v), c(u, v))$ to problem (6.12), (7.13) and (7.20) in $\Omega$. Then the $C^{0}-$ norms of $\alpha$ and $\beta$ have uniform bounds:

(i) If $\theta<\bar{m}$, there holds $2 \theta \leq \alpha-\beta \leq 2 \bar{m}, \alpha \geq \theta, \beta \leq-\theta$;

(ii) If $\theta>\bar{m}$, there holds $2 \bar{m} \leq \alpha-\beta \leq 2 \theta, \alpha \leq \theta, \beta \geq-\theta$. 
Proof. For convenience, let us use system (6.16). For the first case, we construct a region bounded by $L_{1}, L_{2}$ and $L$, as shown in Figure 7.3(a), by Lemma 7.1. Here $L$ is an arbitrary convex curve on the right hand side of $\alpha-\beta=2 \bar{m}$. Note that $L_{1}$ corresponds to $H_{1}$ and $L_{2}$ to $H_{2}$. On the boundary $L_{1}, L_{2}$ of this region, we have

$$
G(\alpha, \beta, c)>0, \quad \text { on } L_{1}, L_{2}
$$

On the other hand, we have

$$
G(\alpha, \beta, c)<0, \quad \text { on } L .
$$

Note that the vector $(\sin \beta,-\cos \beta)$ on $H_{1}$ points toward the interior of $\Omega$, and the vector $(\sin \alpha,-\cos \alpha)$ on $H_{2}$ point towards outside of $\Omega$, see Figure 7.2. We conclude that such a region bounded by $L_{1}, L_{2}$ and $L$ is invariant, see Figure 7.3(a).

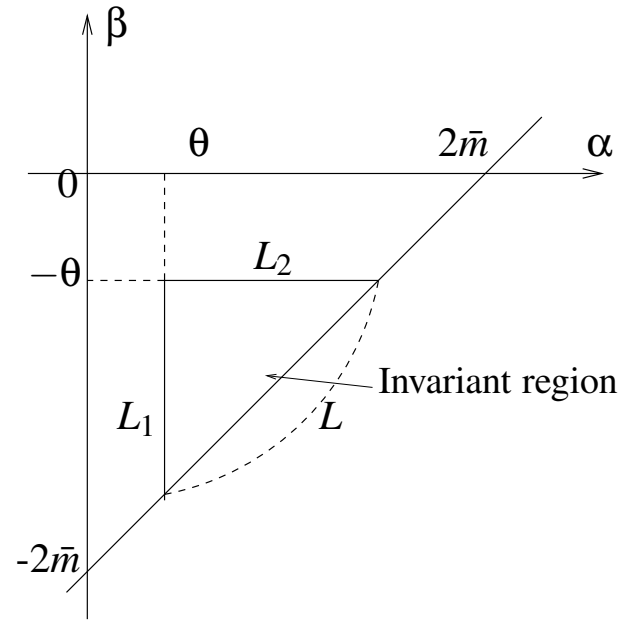

(a) Case $\theta<\bar{m}$

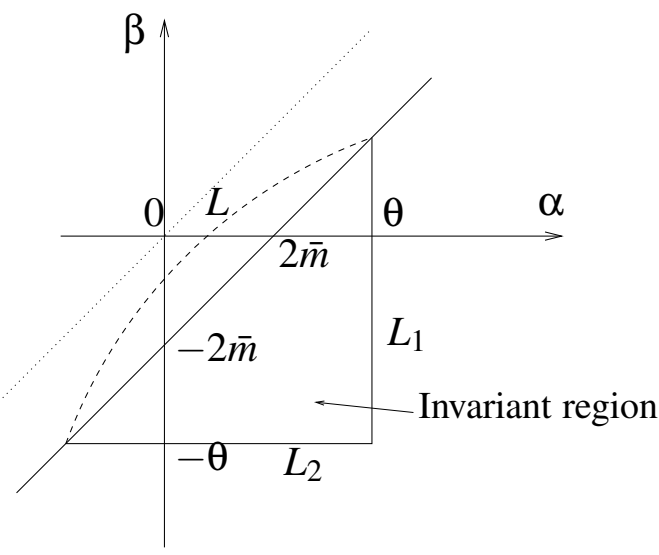

(b) Case $\theta>\bar{m}$

Figure 7.3: Invariant regions

Similarly, we can treat the second case $\theta>\bar{m}$. We construct a region as shown in Figure 7.3(b). If $1<\gamma<3$, the concave curve $L$ is constructed to be located between lines $\alpha-\beta=0$ and $\alpha-\beta=2 \bar{m}$; and if $\gamma \geq 3, L$ is on the left-hand side of $\alpha-\beta=0$. Then we have

$$
\begin{array}{ll}
G(\alpha, \beta, c)<0, & \text { on } L_{1}, L_{2} \\
G(\alpha, \beta, c)>0, & \text { on } L .
\end{array}
$$

Therefore, the region bounded by $L_{1}, L_{2}$ and $L$ is invariant.

By checking the construction of the invariant regions and noting that $L$ is an arbitrary curve, we deduce that $(\alpha, \beta)$ is actually located inside the triangle bounded by $L_{1}, L_{2}$ and the line $\alpha-\beta=2 \bar{m}$. The proof is complete. 
Corollary 7.1. For solutions $(\alpha, \beta, c)$ of (6.16),(7.20) and (7.13), we have:

(i) If $\theta<\bar{m}$, then $G(\alpha, \beta, c)>0$ and $\bar{\partial}_{+} \alpha>0, \bar{\partial}_{-} \beta>0$ for all $(u, v) \in \Omega$.

(ii) If $\theta>\bar{m}$, then $G(\alpha, \beta, c)<0$ and $\bar{\partial}_{+} \alpha<0, \bar{\partial}_{-} \beta<0$ for all $(u, v) \in \Omega$.

Remark 7.1. If the angle of the wedge $\theta$ and the adiabatic index $\gamma$ are related by

$$
\tan ^{2} \theta=\frac{3-\gamma}{\gamma+1}
$$

for $1<\gamma<3$, i.e., $\theta=\bar{m}$, then boundary value (7.20) becomes constant $\left.(\alpha, \beta)\right|_{H_{j}}=(\theta,-\theta)$, $j=1,2$. In this case the invariant region shrinks to a point $(\theta,-\theta)$ on the line $\alpha-\beta=2 \bar{m}$. Note that the source terms of (6.16) vanish on the boundaries $H_{1}, H_{2}$. We can use (6.18) to get an explicit solution,

$$
c=1+\frac{\kappa}{\sin \theta} u,
$$

where $-\sin \theta / \kappa \leq u \leq 0$. We further use (3.13) to get an explicit solution for the original gas expansion problem,

$$
\begin{aligned}
& c=1+\frac{\kappa(\xi \sin \theta-1)}{\kappa+\sin ^{2} \theta}, \\
& u=\frac{\sin \theta(\xi \sin \theta-1)}{\kappa+\sin ^{2} \theta} \\
& v=\eta
\end{aligned}
$$

This solution was first observed in [24].

Remark 7.2. In the proof of Lemma 7.3, we observe that

$$
\frac{\cos ((\alpha+\beta) / 2)}{\sin ((\alpha-\beta) / 2)}>\delta
$$

for some constant $\delta>0$. It follows from the third equation of (6.16) that

$$
c<1+\delta u
$$

for $u<0$ and thus $c$ vanishes at $u>-1 / \delta$. Therefore there exists a curve $u=u(v)$ such that $c(u(v), v)=0$ where $u=u(v)$ is well-defined in the $(u, v)$ plane. This is the interface of gas and vacuum.

Corollary 7.2. For the gas expansion problem, the mappings $(X, Y) \rightarrow(\alpha, \beta)$ and $(X, Y) \rightarrow$ $(A, B)$ are all bijective in the whole region $\Omega$.

Proof. It suffices to check the non-degeneracy of the Jacobian, say from $(X, Y) \rightarrow(\alpha, \beta)$,

$$
J(X, Y ; \alpha, \beta)=-\frac{1}{\sin ^{2}\left(\frac{\alpha-\beta}{2}\right)} \cdot \cot \left(\frac{\alpha-\beta}{2}\right) .
$$

In view of Lemma 7.3, we obtain the conclusion.

Corollary 7.2 show that we can convert system (6.2) into system (6.12) and therefore use system (6.12) or (6.16) to discuss Problem B in the hodograph plane. 


\subsection{Gradient estimates and the proof of Theorem 7.1}

In order to establish the existence of smooth solutions in the whole wave interaction region $\Omega$, we need to establish gradient estimates for system (6.12) or (6.16). Due to the degeneracy of interface $l$, we cut off a sufficient thin strip between the interface $l$ and the level set of $c=\varepsilon$, $\varepsilon>0$. The remaining sub-domain is denoted by $\Omega_{\varepsilon}$, in which $c>\varepsilon$. We first show that there is a unique solution on $\Omega_{\varepsilon}$. Then we extend the solution to $\Omega$ by using the argument of the arbitrariness of $\varepsilon>0$.

Lemma 7.4 (Gradient estimate). Consider system (6.12) or (6.16) with boundary values (7.20) and (7.13). Assume that there is a $C^{1}$ solution $(\alpha, \beta)$ in $\Omega_{\varepsilon}$, then the $C^{1}$ norm of $\alpha$ and $\beta$ has a uniform bound, which only depends on the $C^{0}$ and $C^{1}$ norms of boundary values (7.20). That is, there is a constant $C>0$, depending only the boundary data (7.20) and (7.13), but not on $\varepsilon$, such that

$$
\|(\alpha, \beta)\|_{C^{1}\left(\Omega_{\varepsilon}\right)} \leq C / \varepsilon,
$$

where $\|\cdot\|_{C^{1}\left(\Omega_{\varepsilon}\right)}$ represents the $C^{1}$-norm.

Proof. We use (6.21) to integrate $\bar{\partial}_{-} \alpha$ and $\bar{\partial}_{+} \beta$ along $\lambda_{+}$and $\lambda_{-}-$characteristics, respectively. Noting (6.17), we know that the integral path has a limited length. Also we note that $Q$ has a uniform bound $C / \varepsilon^{2}$ in $\Omega_{\varepsilon}$. Then we deduce that $\bar{\partial}_{-} \alpha$ and $\bar{\partial}_{+} \beta$ are uniformly bounded in $\Omega_{\varepsilon}$,

$$
\left|\bar{\partial}_{-} \alpha\right|<C / \varepsilon, \quad\left|\bar{\partial}_{+} \beta\right|<C / \varepsilon .
$$

On the other hand, since $G$ has a bound $C / \varepsilon$ in $\Omega_{\varepsilon}$ (see (6.16)), so are $\bar{\partial}_{+} \alpha$ and $\bar{\partial}_{-} \beta$,

$$
\left|\bar{\partial}_{+} \alpha\right|<C / \varepsilon, \quad\left|\bar{\partial}_{-} \beta\right|<C / \varepsilon .
$$

Hence using the identities,

$$
\partial_{u}=-\sin ^{-1}(\alpha-\beta)\left(\cos \alpha \bar{\partial}_{+}-\cos \beta \bar{\partial}_{-}\right), \quad \partial_{v}=-\sin ^{-1}(\alpha-\beta)\left(\sin \alpha \bar{\partial}_{+}-\sin \beta \bar{\partial}_{-}\right),
$$

and using the hyperbolicity $\alpha \neq \beta$ in $\Omega_{\varepsilon}$, we conclude that $\partial_{u} \alpha, \partial_{\nu} \alpha, \partial_{u} \beta$ and $\partial_{\nu} \beta$ are uniformly bounded in $\Omega_{\varepsilon}$, as expressed in (7.37).

Lemma 7.5 (Modulus estimate). Assume that the solution $(\alpha, \beta) \in C^{1}\left(\Omega_{\varepsilon}\right)$. Then we have the following modulus estimate,

$$
\|(\alpha, \beta)\|_{C^{1,1}\left(\Omega_{\varepsilon}\right)}<C / \varepsilon^{2}
$$

where $\|\cdot\|_{C^{1,1}\left(\Omega_{\varepsilon}\right)}$ represents the $C^{1,1}$-norm, and $C^{1,1}\left(\Omega_{\varepsilon}\right)$ is the space of functions whose $C^{1}$-derivatives are Lipschitz continuous.

Proof. Using (6.21), we follow [7] or [17] to obtain

$$
\left\|\bar{\partial}_{-} \alpha\right\|_{C^{1,1}\left(\Omega_{\varepsilon}\right)}<C / \varepsilon^{2}, \quad\left\|\bar{\partial}_{+} \beta\right\|_{C^{1,1}\left(\Omega_{\varepsilon}\right)}<C / \varepsilon^{2} .
$$


Then we use the same approach to derive

$$
\left\|\bar{\partial}_{+} \alpha\right\|_{C^{1,1}\left(\Omega_{\varepsilon}\right)}<C / \varepsilon^{2}, \quad\left\|\bar{\partial}_{-} \beta\right\|_{C^{1,1}\left(\Omega_{\varepsilon}\right)}<C / \varepsilon^{2} .
$$

Thus the identities (7.40) are used to yield (7.41).

Proof of Theorem 7.1. With the classical technique in [17] or [7], we obtain the "global" solution in $\Omega_{\varepsilon}$ by the extension from the local solution.

In view of Lemma 7.2, we obain a local solution $(\alpha, \beta, c)$ in $\Omega_{\delta}=\left\{(u, v) \in \Omega_{\varepsilon} ;-\delta<u<0\right\}$. We take a level set of $c$, denoted by $\Upsilon_{c}$, in $\Omega_{\delta}$. On this curve, $(\alpha, \beta, c)$ is known from the local solution and $(\alpha, \beta) \in C^{1}\left(\Upsilon_{c}\right)$ in view of Lemma 7.5. Then our problem becomes to find a solution of (6.12) in the remaining region, subject to the data on $H_{1}, H_{2}$ and $\Upsilon_{c}$.

Denote the slope of $\Upsilon_{c}$ by $s_{0}$,

$$
s_{0}:=\frac{d v}{d u}=-\frac{c_{u}}{c_{v}}=-\cot \left(\frac{\alpha+\beta}{2}\right) .
$$

Then we have

$$
\frac{1}{s_{0}}-\frac{1}{\lambda_{-}}=\frac{\sin \left(\frac{\alpha-\beta}{2}\right)}{\cos \left(\frac{\alpha+\beta}{2}\right) \cos \alpha}>0, \frac{1}{s_{0}}-\frac{1}{\lambda_{+}}=-\frac{\sin \left(\frac{\alpha-\beta}{2}\right)}{\cos \left(\frac{\alpha+\beta}{2}\right) \cos \beta}<0 .
$$

This shows that the level set $\Upsilon_{c}$ is not a characteristic and $\lambda_{ \pm}$-characteristics always points toward the right hand side of $\Upsilon_{c}$. Thus, we follow the proof of Lemma 4.1 in [7, Page 294], using Lemmas 7.4 and 7.5, to finish the proof of the existence of solutions in $\Omega_{\varepsilon}$.

Owing to the arbitrariness of width $\varepsilon>0$, we use the contradiction argument to show that the $C^{1}$ solution $(\alpha, \beta, c)$ can be extend to the whole region $\Omega$.

The discussion of vacuum boundary is left in Subsection 7.7.2.

\subsection{Inversion}

We now discuss the inversion of the hodograph transform, i.e., the Jacobian $J_{T}^{-1}(u, v ; \xi, \eta)$ in (3.19) does not vanish for the gas expansion problem. Before doing this, we investigate the curvature of $\lambda_{ \pm}$-characteristics. In other words, we need precise estimates on the gradient of the variables $\alpha, \beta$.

Using the evolution equations for $\bar{\partial}_{-} \alpha$ and $\bar{\partial}_{+} \beta$ in Theorem 6.2, we can obtain preliminary (rather rough) gradient estimates on $\alpha$ and $\beta$. 
Lemma 7.6 (Preliminary gradient estimates). Consider the solution $(\alpha, \beta, c)$ of $(6.16)$ and (7.20) for the gas expansion problem. There are two categories: (I) $\gamma \geq 2$ and (II) $1<\gamma<2$. For each, there are three sub-categories. They are stated as follows. See Figure 7.4.

$\left(I_{1}\right)$. If $2 \leq \gamma<3$ and $\theta \leq \bar{m}(\leq \pi / 6)$, then

$$
\bar{\partial}_{-} \alpha \leq 0, \quad \text { and } \quad \bar{\partial}_{+} \beta \leq 0 .
$$

(I2). If $\gamma \geq 2$ and $\bar{m}<\theta \leq \pi / 6$, we have

$$
\bar{\partial}_{-} \alpha \geq 0, \text { and } \bar{\partial}_{+} \beta \geq 0
$$

(I $\left.I_{3}\right)$. If $\gamma \geq 2$ and $\pi / 6<\theta<\pi / 2$, the signs of $\bar{\partial}_{-} \alpha$ and $\bar{\partial}_{+} \beta$ are not definite. That is, the convexity of $\lambda_{ \pm}$may change.

(II $)$ If $1<\gamma<2$ and $\bar{m} \leq \theta<\pi / 2$, then

$$
\bar{\partial}_{-} \alpha \leq 0, \quad \text { and } \bar{\partial}_{+} \beta \leq 0 \text {. }
$$

(II $)$ If $1<\gamma<2$ and $\pi / 6<\theta<\bar{m}$, then

$$
\bar{\partial}_{-} \alpha \geq 0, \text { and } \bar{\partial}_{+} \beta \geq 0 \text {. }
$$

$\left(I_{3}\right)$. If $1<\gamma<2$ and $0<\theta<\pi / 6$, the signs of $\bar{\partial}_{-} \alpha$ and $\bar{\partial}_{+} \beta$ are not definite. That is, the convexity of $\lambda_{ \pm}$may change.

We note that these two categories are parallel and symmetric.

Proof. We use (6.21) and check the sign of $Q$ for each case. By noting that $\left.\bar{\partial}_{-} \alpha\right|_{H_{1}}=0$ and $\left.\bar{\partial}_{+} \beta\right|_{H_{2}}=0$, we immediately prove this lemma.

Now we look at the hodograph transformation $T:(\xi, \eta) \rightarrow(u, v)$. The mapping (3.13) defines a domain via $\xi=u+i_{u}, \eta=v+i_{v}$. We need to show that no two points map to one,

$$
J_{T}^{-1}(u, v ; \xi, \eta)=\xi_{u} \eta_{v}-\xi_{v} \eta_{u}=\left(1+i_{u u}\right)\left(1+i_{v v}\right)-i_{u v}^{2} \neq 0 .
$$

We calculate, on the one hand, using (3.16),

$$
\left(2 \kappa i-i_{u}^{2}\right) i_{u v}^{2}+2 i_{u} i_{v} i_{u v}\left(1+i_{u u}\right)+\left(2 \kappa i-i_{v}^{2}\right)\left(1+i_{u u}\right)^{2}=\left(2 \kappa i-i_{u}^{2}\right)\left[i_{u v}^{2}-\left(1+i_{u u}\right)\left(1+i_{v v}\right)\right] .
$$

On the other hand, we have

$$
\left(2 \kappa i-i_{u}^{2}\right) i_{u v}^{2}+2 i_{u} i_{v} i_{u v}\left(1+i_{u u}\right)+\left(2 \kappa i-i_{v}^{2}\right)\left(1+i_{u u}\right)^{2}=\left(2 \kappa i-i_{v}^{2}\right)\left(\partial_{+} i_{u}+1\right)\left(\partial_{-} i_{u}+1\right) .
$$




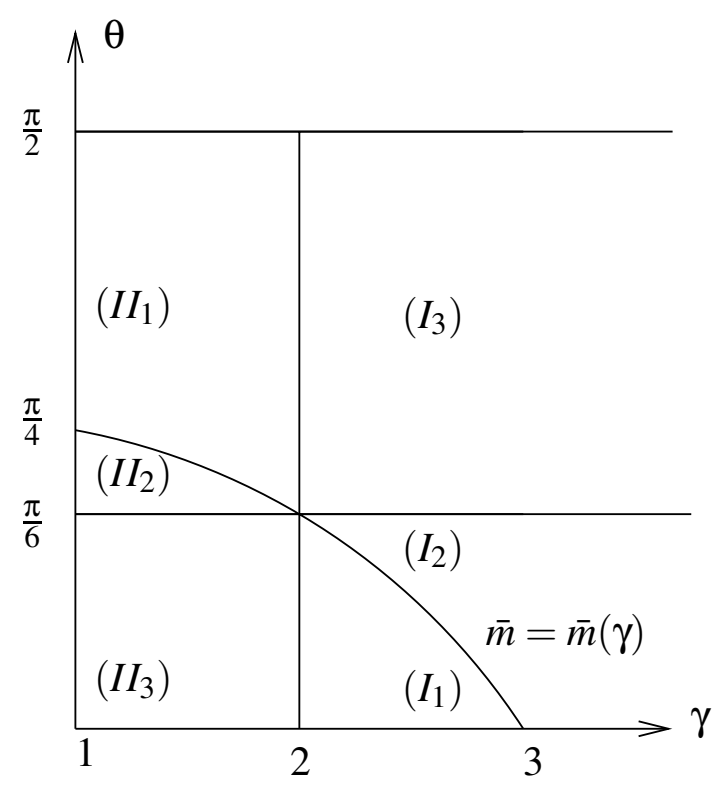

Figure 7.4: Ranges of gas constant $\gamma$ and wedge half angle $\theta$

Then we obtain

$$
J_{T}^{-1}(u, v ; \xi, \eta)=-\frac{1}{\lambda_{-} \lambda_{+}}\left(\partial_{+} X+1\right)\left(\partial_{-} X+1\right)=\frac{1}{\cos \alpha \cos \beta}\left(\bar{\partial}_{+} X+\sin \beta\right)\left(\bar{\partial}_{-} X+\sin \alpha\right),
$$

by using the definition of $\bar{\partial}_{ \pm}$, see (6.15). This is parallel to (5.5). Therefore, in order to show that $J_{T}^{-1}(u, v ; \xi, \eta)$ does not vanish, it is equivalent to prove that:

Lemma 7.7. The non-degeneracy of the Jacobian $J_{T}^{-1}(u, v ; \xi, \eta)$ is equivalent to

$$
\bar{\partial}_{+} X+\sin \beta \neq 0 \text { and } \bar{\partial}_{-} X+\sin \alpha \neq 0 \text {. }
$$

Recall the expression of $X$ in terms of $\alpha, \beta$ in (6.11). Then we compute

$$
\begin{aligned}
\bar{\partial}_{+} X+\sin \beta= & -\kappa \frac{\cos \left(\frac{\alpha+\beta}{2}\right)}{\sin \left(\frac{\alpha-\beta}{2}\right)}-\frac{1+\kappa}{2} \cot \left(\frac{\alpha-\beta}{2}\right) \cos \beta\left[\tan ^{2} \bar{m}-\tan ^{2}\left(\frac{\alpha-\beta}{2}\right)\right] \\
& +\sin \beta+\frac{c}{2} \frac{\cos \alpha}{\sin ^{2}\left(\frac{\alpha-\beta}{2}\right)} \bar{\partial}_{+} \beta \\
\bar{\partial}_{-} X+\sin \alpha= & \kappa \frac{\cos \left(\frac{\alpha+\beta}{2}\right)}{\sin \left(\frac{\alpha-\beta}{2}\right)}+\frac{1+\kappa}{2} \cot \left(\frac{\alpha-\beta}{2}\right) \cos \alpha\left[\tan ^{2} \bar{m}-\tan ^{2}\left(\frac{\alpha-\beta}{2}\right)\right] \\
& +\sin \alpha-\frac{c}{2} \frac{\cos \beta}{\sin ^{2}\left(\frac{\alpha-\beta}{2}\right)} \bar{\partial}_{-} \alpha .
\end{aligned}
$$


They are easily simplified to be

$$
\begin{aligned}
\bar{\partial}_{+} X+\sin \beta & =-\frac{1+\kappa}{\sin (\alpha-\beta)} \cos \alpha+\frac{c}{2} \frac{\cos \alpha}{\sin ^{2}\left(\frac{\alpha-\beta}{2}\right)} \bar{\partial}_{+} \beta \\
& =\frac{c}{2} \frac{\cos \alpha}{\sin ^{2}\left(\frac{\alpha-\beta}{2}\right)}\left[\bar{\partial}_{+} \beta-\frac{1+\kappa}{c} \tan \left(\frac{\alpha-\beta}{2}\right)\right] \\
\bar{\partial}_{-} X+\sin \alpha & =\frac{1+\kappa}{\sin (\alpha-\beta)} \cos \beta-\frac{c}{2} \frac{\cos \beta}{\sin ^{2}\left(\frac{\alpha-\beta}{2}\right)} \bar{\partial}_{-} \alpha \\
& =-\frac{c}{2} \frac{\cos \beta}{\sin ^{2}\left(\frac{\alpha-\beta}{2}\right)}\left[\bar{\partial}_{-} \alpha-\frac{1+\kappa}{c} \tan \left(\frac{\alpha-\beta}{2}\right)\right] .
\end{aligned}
$$

Note that on the boundary $H_{1}, H_{2}$, the values $\bar{\partial}_{+} \beta$ and $\bar{\partial}_{-} \alpha$ are, respectively,

$$
\left.\bar{\partial}_{+} \beta\right|_{H_{2}} \equiv 0,\left.\quad \bar{\partial}_{-} \alpha\right|_{H_{1}} \equiv 0 \text {. }
$$

Therefore (7.54) follows from the following Lemma.

Lemma 7.8. There holds

$$
\bar{\partial}_{+} \beta<\frac{1+\kappa}{c} \tan \left(\frac{\alpha-\beta}{2}\right), \quad \bar{\partial}_{-} \alpha<\frac{1+\kappa}{c} \tan \left(\frac{\alpha-\beta}{2}\right)
$$

in the region $\Omega$, see Figure 7.2.

In order to prove this lemma, we need to use:

Lemma 7.9. Consider the following initial value problem for ODEs,

$$
\left\{\begin{array}{l}
\frac{d y}{d x}+P(x, y) y=R(x, y), \quad x>0 \\
y(0)=0
\end{array}\right.
$$

where $P(x, y), R(x, y)>0$. If $\frac{d}{d x}[R(x, y(x)) / P(x, y(x))]>0$, then the solution $y=y(x)$ is bounded by the asymptotic state $R / P$,

$$
0 \leq y(x)<R / P, \quad \forall x>0 .
$$

Proof. The proof is simple. Note that (7.60) holds for $0<x<\delta$ for some small $\delta>0$. Set

$$
x_{0}=\min \{x>0 ; y(x)=R(x, y(x)) / P(x, y(x))\} .
$$

Then at this point we have

$$
\begin{aligned}
\frac{d}{d x}(P y-R) & =\frac{d P}{d x} \cdot y+P \frac{d y}{d x}-\frac{d R}{d x} \\
& =\frac{d P}{d x} \cdot \frac{R}{P}-\frac{d R}{d x} \\
& <0,
\end{aligned}
$$

by using the condition on $P$ and $R$. This contradicts that $P y-R$ cannot attain the zero value from the left hand side of $x_{0}$. 
Proof of Lemma 7.8. Now we prove (7.58) case by case, in accordance with Lemma 7.6.

$\left(I_{1}\right) 2 \leq \gamma<3$ and $\theta \leq \bar{m}$. We have $\bar{\partial}_{-} \alpha \leq 0$ and $\bar{\partial}_{+} \beta \leq 0$ in view of Lemma 7.6. Thus (7.58) holds obviously.

( $\left.I_{2}\right) \gamma \geq 2$ and $\bar{m} \leq \theta<\pi / 6$. This is the most delicate and representative case, for which we have

$$
\tan ^{2} \bar{m} \leq \tan ^{2}\left(\frac{\alpha-\beta}{2}\right)<1 / 3
$$

Set

$$
S:=\frac{1+\kappa}{2 c} \sin (\alpha-\beta)\left[1-\tan ^{2}\left(\frac{\alpha-\beta}{2}\right)\right] .
$$

We want to use Lemma 7.9 to show that $S$ is the upper bound of both $\bar{\partial}_{-} \alpha$ and $\bar{\partial}_{+} \beta$,

$$
\bar{\partial}_{-} \alpha<S, \quad \bar{\partial}_{+} \beta<S .
$$

Since $S<\frac{1+\kappa}{c} \tan \left(\frac{\alpha-\beta}{2}\right)$, the inequalities (7.58) follow immediately.

Since $\bar{\partial}_{-} \alpha>0$ and $\bar{\partial}_{+} \beta>0$ inside $\Omega$ in view of Lemma 7.6 as well as $\left.\bar{\partial}_{-} \alpha\right|_{H_{1}}=\left.\bar{\partial}_{+} \beta\right|_{H_{2}} \equiv 0$, we claim that

$$
\bar{\partial}_{-} S>0, \quad \bar{\partial}_{+} S>0,
$$

provided that (7.65) holds. Indeed, we compute

$$
\begin{aligned}
-\bar{\partial}_{-} S= & \frac{(1+\kappa) \kappa}{2 c^{2}} \sin (\alpha-\beta)\left[1-\tan ^{2}\left(\frac{\alpha-\beta}{2}\right)\right] \\
& -\frac{1+\kappa}{2 c}\left[\cos (\alpha-\beta)\left(1-\tan ^{2}\left(\frac{\alpha-\beta}{2}\right)\right)-2 \tan ^{2}\left(\frac{\alpha-\beta}{2}\right)\right]\left(\bar{\partial}_{-} \alpha-\bar{\partial}_{-} \beta\right) .
\end{aligned}
$$

Note again that $\bar{\partial}_{-} \alpha>0, \bar{\partial}_{-} \beta<0$ and thus $\bar{\partial}_{-} \alpha-\bar{\partial}_{-} \beta>0$ in $\Omega$ for the present case. So if

$$
\cos (\alpha-\beta)\left(1-\tan ^{2}\left(\frac{\alpha-\beta}{2}\right)\right)-2 \tan ^{2}\left(\frac{\alpha-\beta}{2}\right)<0,
$$

then (7.66) holds obviously. Otherwise, we have, if (7.65) hold,

$$
\bar{\partial}_{-} \alpha-\bar{\partial}_{-} \beta<\frac{1+\kappa}{2 c} \sin (\alpha-\beta)\left(1-\tan ^{2} \bar{m}\right)=\frac{\kappa}{c} \sin (\alpha-\beta) .
$$

It follows that

$$
\begin{aligned}
-\bar{\partial}_{-} S & >\frac{(1+\kappa) \kappa}{2 c^{2}} \sin (\alpha-\beta)\left[\left(1-\tan ^{2}\left(\frac{\alpha-\beta}{2}\right)\right)(1-\cos (\alpha-\beta))+2 \tan ^{2}\left(\frac{\alpha-\beta}{2}\right)\right] \\
& >0 .
\end{aligned}
$$

Similarly we can prove $\bar{\partial}_{+} S>0$. 
We further note that

$$
0<Q / W<S
$$

In fact, we compute

$$
\begin{gathered}
Q-S W=\frac{1+\kappa}{c} \sin (\alpha-\beta) \tan ^{2}\left(\frac{\alpha-\beta}{2}\right) \\
\cdot\left\{\left[\tan ^{2} \bar{m}-\tan ^{2}\left(\frac{\alpha-\beta}{2}\right)\right]\left[3 \tan ^{2}\left(\frac{\alpha-\beta}{2}\right)-1\right] \cos ^{2}\left(\frac{\alpha-\beta}{2}\right)-\left[1-\tan ^{2}\left(\frac{\alpha-\beta}{2}\right)\right]\right\} .
\end{gathered}
$$

The quantity in the brace is negative for the present case. So, (7.71) holds. Denote

$$
\widetilde{W}:=Q / S .
$$

Then we have

$$
0 \leq \widetilde{W}<W .
$$

Construct the initial value problem for a system of the following system of ODEs,

$$
\begin{aligned}
\bar{\partial}_{+} \phi+\widetilde{W} \phi & =Q, \\
-\bar{\partial}_{-} \psi+\widetilde{W} \psi & =Q,
\end{aligned}
$$

with zero boundary values,

$$
\left.\phi\right|_{H_{1}} \equiv 0,\left.\quad \psi\right|_{H_{2}} \equiv 0 .
$$

By the comparison principle, we conclude

$$
\bar{\partial}_{-} \alpha<\phi, \quad \bar{\partial}_{+} \beta<\psi,
$$

inside the domain $\Omega$.

We claim that

$$
\phi<S, \quad \psi<S,
$$

inside $\Omega$. Indeed, note that the vector fields $\bar{\partial}_{+}$on $H_{1}$ and $-\bar{\partial}_{-}$all point towards the interior of $\Omega$. Suppose on the contrary that there is a "maximum" point $\left(u_{0}, v_{0}\right)$ in $\Omega$ such that at this point $\phi=S$ and $\psi<S$ for all $u_{0}<u \leq 0$, i.e.,

$$
u_{0}=\max \{u ; \phi(u, v)=S(\alpha(u, v), \beta(u, v)) \text { and } \psi(u, v)<S(\alpha(u, v), \beta(u, v))\} .
$$

At $\left(u_{0}, v_{0}\right)$, we know $\bar{\partial}_{-} \alpha<\phi=S$ and $\bar{\partial}_{+} \beta<\psi<S$ by using (7.77). Then in view of Lemma 7.9 , we deduce $\phi<S$ at $\left(u_{0}, v_{0}\right)$, which yields a contradiction.

Thus we have shown that (7.78) holds and so does (7.65). The proof of Case $\left(I_{2}\right)$ is complete.

$\left(I_{3}\right) . \gamma \geq 2$ and $\pi / 6<\theta<\pi / 2$. The proof of this case follows from Cases $\left(I_{1}\right)$ and $\left(I_{2}\right)$. First, when the solution $(\alpha, \beta)$ is in the region $(\alpha-\beta) / 2>\pi / 6$ (see Figure 7.3), we have

$$
\tan ^{2}\left(\frac{\alpha-\beta}{2}\right)>1 / 3>\tan ^{2} \bar{m}
$$


It follows that $\bar{\partial}_{-} \alpha<0$ and $\bar{\partial}_{+} \beta<0$ in this region. Then the solution $(\alpha, \beta)$ enters the region $\bar{m}<(\alpha-\beta) / 2<\pi / 6$, in which $(\alpha, \beta)$ satisfies

$$
\tan ^{2} \bar{m}<\tan ^{2}\left(\frac{\alpha-\beta}{2}\right)<1 / 3
$$

and $\bar{\partial}_{-} \alpha$ and $\bar{\partial}_{+} \beta$ become increasing from negative values, along $\bar{\partial}_{-}$and $-\bar{\partial}_{-}$, respectively. If $\bar{\partial}_{-} \alpha$ and $\bar{\partial}_{+} \beta$ never attain zeroes, (7.58) holds automatically. Otherwise, we use the same argument as in Case $\left(I_{2}\right)$ from the point at which $\bar{\partial}_{-} \alpha=0$ or $\bar{\partial}_{+} \beta=0$. The convexity of $\lambda_{ \pm}$-characteristics, or the sign change of $\bar{\partial}_{-} \alpha$ and $\bar{\partial}_{+} \beta$, is illustrated in Figure 7.5. Thus we prove Lemma 7.8 for this case.

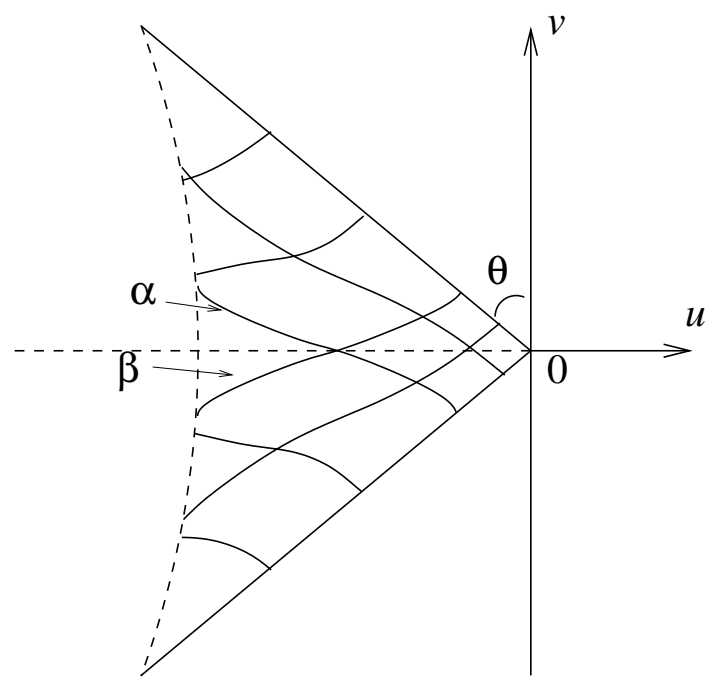

Figure 7.5: Changes of convexity types of $\lambda_{ \pm}$-characteristics

For the second big category $(I I)$ that $1<\gamma<2$, we do the proof parallel to $(I)$ case by case. Specifically, we have

$\left(I I_{1}\right)$. If $1<\gamma<2$ and $\theta>\bar{m}$, there holds,

$$
\bar{\partial}_{-} \alpha \leq 0, \quad \bar{\partial}_{+} \beta \leq 0 .
$$

$\left(I I_{2}\right)$. If $1<\gamma<2$ and $\pi / 6 \leq \theta \leq \bar{m}$, there holds,

$$
\begin{aligned}
& 0 \leq \bar{\partial}_{-} \alpha<\frac{1+\kappa}{2 c} \sin (\alpha-\beta)\left[1-\tan ^{2}\left(\frac{\alpha-\beta}{2}\right)\right], \\
& 0 \leq \bar{\partial}_{+} \beta<\frac{1+\kappa}{2 c} \sin (\alpha-\beta)\left[1-\tan ^{2}\left(\frac{\alpha-\beta}{2}\right)\right] .
\end{aligned}
$$

$\left(I I_{3}\right)$. If $1<\gamma<2$ and $0<\theta<\pi / 6, \bar{\partial}_{-} \alpha$ and $\bar{\partial}_{+} \beta$ may changes signs, but their upper bounds in (7.83) are still valid. 
The proof of Lemma 7.8 is complete.

\subsection{Proof of Theorem 7.2}

The above estimates are sufficient for the proof of Theorem 7.2. For completeness, we sum it as follows. First we use the hodograph transformation (3.11) to convert Problem A into Problem B. Since the region $\mathcal{D}$ in Figure 7.1(b) is a wave interaction region, the Jacobian $J_{T}(u, v ; \xi, \eta)$ does not vanish in view of Theorem 5.1, so the hodograph transformation (3.11) is valid. Then we solve Problem $B$ in Theorem 7.1. In Lemmas 7.8 and 7.7, we show that the hodograph transformation $J_{T}(u, v ; \xi, \eta)$ is invertible. Thus the proof of Theorem 7.2 is complete.

\subsection{Properties of the solutions}

\subsubsection{Convexity of characteristics in the physical plane}

Now we discuss the convexity of $\Lambda_{ \pm}$-characteristics in the mixed wave region $\mathcal{D}$, in the $(\xi, \eta)$ plane. It is a rather simple way to look at this from the correspondence between the $(\xi, \eta)$ plane and the $(u, v)$ plane.

Consider the hodograph transformation $T$ of (3.11). We note, by using the chain rule, that,

$$
\frac{\partial}{\partial u}+\lambda_{+} \frac{\partial}{\partial v}=\left(\frac{\partial \xi}{\partial u}+\lambda_{+} \frac{\partial \xi}{\partial v}\right) \frac{\partial}{\partial \xi}+\left(\frac{\partial \eta}{\partial u}+\lambda_{+} \frac{\partial \eta}{\partial v}\right) \frac{\partial}{\partial \eta}
$$

We rewrite (3.14) as

$$
\frac{\partial \xi}{\partial u}+\lambda_{+} \frac{\partial \xi}{\partial v}=-\lambda_{-}\left(\frac{\partial \eta}{\partial u}+\lambda_{+} \frac{\partial \eta}{\partial v}\right)
$$

Using (3.13), we have

$$
\frac{\partial \xi}{\partial u}+\lambda_{+} \frac{\partial \xi}{\partial v}=\partial_{+} X+1
$$

Thus we derive a differential relation from (7.84), by noting $\Lambda_{+}=-1 / \lambda_{-}$,

$$
\bar{\partial}_{+}=\left(\bar{\partial}_{+} X+\sin \beta\right)\left(\frac{\partial}{\partial \xi}+\Lambda_{+} \frac{\partial}{\partial \eta}\right)
$$

Similarly, we have

$$
\bar{\partial}_{-}=\left(\bar{\partial}_{-} X+\sin \alpha\right)\left(\frac{\partial}{\partial \xi}+\Lambda_{-} \frac{\partial}{\partial \eta}\right)
$$


Acting (7.87) on $\Lambda_{+}$and (7.88) on $\Lambda_{-}$as well as using the definition of $\alpha, \beta$ (i.e., $\Lambda_{+}=\tan \alpha$, $\left.\Lambda_{-}=\tan \beta\right)$, we obtain

$$
\begin{aligned}
& \left(\frac{\partial}{\partial \xi}+\Lambda_{+} \frac{\partial}{\partial \eta}\right) \Lambda_{+}=\frac{1}{\cos ^{2} \alpha} \cdot\left(\bar{\partial}_{+} X+\sin \beta\right)^{-1} \cdot \bar{\partial}_{+} \alpha \\
& \left(\frac{\partial}{\partial \xi}+\Lambda_{-} \frac{\partial}{\partial \eta}\right) \Lambda_{-}=\frac{1}{\cos ^{2} \beta} \cdot\left(\bar{\partial}_{-} X+\sin \alpha\right)^{-1} \cdot \bar{\partial}_{-} \beta
\end{aligned}
$$

Therefore, the convexity of $\Lambda_{ \pm}$-characteristics is determined by two factors, respectively. By Corollary 7.1, the signs of $\bar{\partial}_{+} \alpha$ and $\bar{\partial}_{-} \beta$ just depend on the relation between the wedge angle $\theta$ and the index $\bar{m}$, i.e.,

$$
\bar{\partial}_{+} \alpha<0, \quad \bar{\partial}_{-} \beta<0,
$$

if $\theta>\bar{m} ;$ and

$$
\bar{\partial}_{+} \alpha>0, \quad \bar{\partial}_{-} \beta>0,
$$

if $\theta<\bar{m}$. In view of Lemma 7.8, we have

$$
\bar{\partial}_{+} X+\sin \beta<0, \quad \bar{\partial}_{-} X+\sin \alpha>0 .
$$

Hence we conclude,

$$
\left(\frac{\partial}{\partial \xi}+\Lambda_{+} \frac{\partial}{\partial \eta}\right) \Lambda_{+}>0,\left(\frac{\partial}{\partial \xi}+\Lambda_{-} \frac{\partial}{\partial \eta}\right) \Lambda_{-}<0, \text { for } \theta>\bar{m}
$$

and

$$
\left(\frac{\partial}{\partial \xi}+\Lambda_{+} \frac{\partial}{\partial \eta}\right) \Lambda_{+}<0, \quad\left(\frac{\partial}{\partial \xi}+\Lambda_{-} \frac{\partial}{\partial \eta}\right) \Lambda_{-}>0, \text { for } \theta<\bar{m} .
$$

Theorem 7.3. The $\Lambda_{ \pm}$-characteristics in the wave interaction region $\mathcal{D}$ of $(\xi, \eta)$ plane have fixed convexity types:

(i) If $\theta>\bar{m}$, the $\Lambda_{+}$-characteristics are convex and the $\Lambda_{-}$-characteristics are concave.

(ii) If $\theta<\bar{m}$, the $\Lambda_{+}$-characteristics are concave and the $\Lambda_{-}$-characteristics are convex.

(iii) If $\theta=\bar{m}$, the solution has the explicit form (7.32) and all characteristics are straight.

\subsubsection{Regularity of the vacuum boundary.}

Recall that the formulae (3.13) transform the solution $(\alpha, \beta, c)$ in the $(u, v)$ plane, back into the $(\xi, \eta)$-plane. Note that $(\alpha, \beta)$, and thus $c_{u}, c_{v}$, are uniformly bounded for $1<\gamma<3$, and that $c$ tends to zero with a rate much faster than $c_{u}, c_{v}$ for $\gamma \geq 3$. We conclude that on the vacuum boundary, the $(u, v)$ coordinates coincide with the $(\xi, \eta)$ coordinates. This is because, by using (3.13),

$$
\xi=u+i_{u}=u+\frac{c}{\kappa} c_{u}=u, \quad \eta=v+i_{v}=v+\frac{c}{\kappa} c_{v}=v .
$$




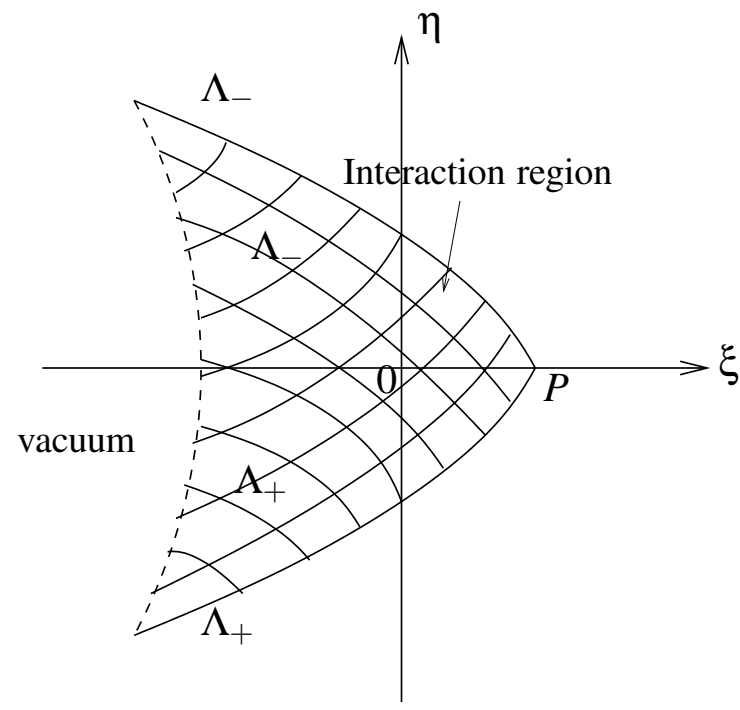

(a) Case $\theta>\bar{m}$

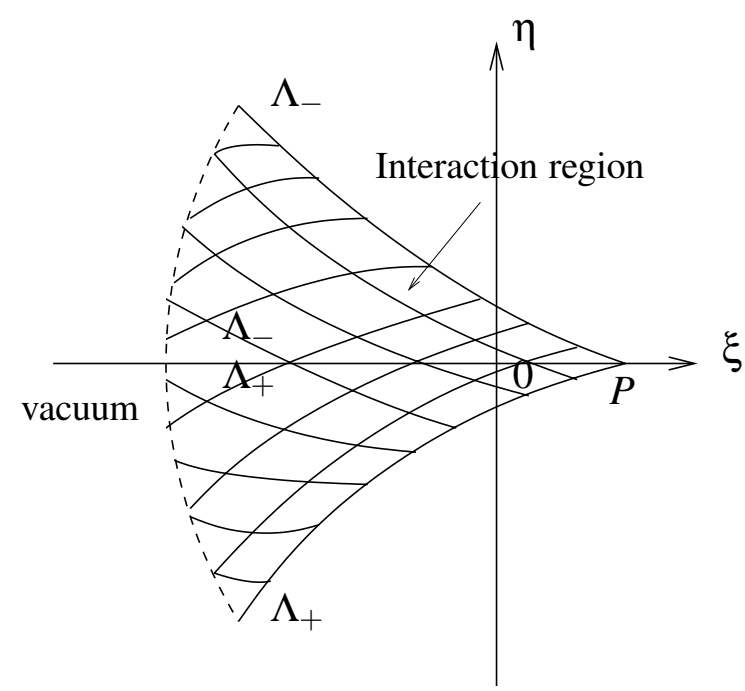

(b) Case $\theta<\bar{m}$

Figure 7.6: Convexity types of the characteristics and the vacuum boundaries in the $(\xi, \eta)$ plane are opposite to each other in the two cases.

We prove that the vacuum boundary is Lipschitz continuous. Let us consider the curve $\{(u, v) \mid i(u, v)=\varepsilon>0\}$ for all small positive $\varepsilon$. Differentiating the equation $i(u(v), v)=\varepsilon$ with respect to $v$, we find

$$
\frac{d u}{d v}=-\frac{Y}{X}=-\tan \left(\frac{\alpha+\beta}{2}\right) .
$$

Since $|\alpha+\beta|<\pi / 2$ uniformly with respect to $\varepsilon>0$, the level curve $i(u, v)=\varepsilon$ has a bounded derivative and in the limit as $\varepsilon \rightarrow 0+$ converges to a Lipschitz continuous vacuum boundary.

\subsubsection{Relative location}

For the explicit solution when $\theta=\bar{m}$, the vacuum boundary is a vertical segment. Now we hold $\theta$ fixed and consider varying $\gamma$ so that $\theta<\bar{m}$. Then we find $\alpha$ and $\beta$ lies on the left-hand side of the line $\alpha-\beta=2 \bar{m}$ in the $\alpha-\beta$ phase plane. By the formula $i_{v}=Y$ and the location of the boundary data, we have $Y<0$ on the upper half of the wedge, thus $i$ is monotone decreasing in $v$ on the upper half, hence the vacuum boundary is on the left of the Suchkov boundary and of a concave type. Similarly, the other case $\theta>\bar{m}$ has the opposite result.

Theorem 7.4. Let the vacuum boundary be represented as $\xi=\xi(\eta)$. Then it is Lipschitz continuous. It is less than the Suchkov solution boundary and is convex if $\theta<\bar{m}$, but it is concave and greater than the Suchkov solution boundary for $\theta>\bar{m}$. 


\subsubsection{Characteristics on the vacuum boundary}

We already know that the sound speed $c$ attains zero in a finite range of $u$. Conversely, from (6.17) we deduce that the lengths of $\lambda_{ \pm}$-characteristics are limited. Then in view of (6.16) it can be seen that on the vacuum boundary,

$$
\alpha-\beta=2 \bar{m} .
$$

As a matter of fact, on one hand, if (7.97) would not be true, then $\bar{\partial}_{+} \alpha$ and $\bar{\partial}_{-} \beta$ become infinity as $(u, v)$ is close to the vacuum boundary, which forces $(\alpha, \beta)$ to reach the line $\alpha-\beta=2 \bar{m}$ in the $(\alpha, \beta)$-plane. See Figure 7.3. On the other hand, the line $\alpha-\beta=2 \bar{m}$ is the set of stationary points of $(\alpha, \beta)$. Thus once (7.97) holds, we have $c=0$.

Thus we see clearly the distribution of characteristics on the vacuum boundary. In particular, for $1<\gamma<3$, a $\Lambda_{+}$-characteristic line has a non-zero intersection angle with a $\Lambda_{-}-$ characteristic line. However, if $\gamma \geq 3$, we have $\alpha=\beta$ on the vacuum boundary such that they are all tangent to the vacuum boundary.

\section{Summary remarks}

We have considered the phase space equation (3.15)

$$
\left(2 \kappa i-i_{u}^{2}\right) i_{v v}+2 i_{u} i_{v} i_{u v}+\left(2 \kappa i-i_{v}^{2}\right) i_{u u}=i_{u}^{2}+i_{v}^{2}-4 \kappa i
$$

known from 1958 for the enthalpy $i$ with the inverse of the hodograph transformation (3.13)

$$
\xi=u+i_{u}, \quad \eta=v+i_{v}
$$

for the two-dimensional self-similar isentropic ir-rotational Euler system. Upon introducing the variables of inclination angles of characteristics and normalized characteristic derivatives, we have changed the second-order phase space equation to a first order system (6.16)

$$
\bar{\partial}_{+} \alpha=G(\alpha, \beta, c), \quad \bar{\partial}_{-} \beta=G(\alpha, \beta, c), \quad \partial_{0} c=\kappa \cos \left(\frac{\alpha+\beta}{2}\right) / \sin \left(\frac{\alpha-\beta}{2}\right),
$$

where

$$
G(\alpha, \beta, c)=\frac{1+\kappa}{2 c} \cdot \sin (\alpha-\beta) \cdot\left[m-\tan ^{2} \frac{\alpha-\beta}{2}\right] .
$$

Derivatives of the variables $\alpha$ and $\beta$ along directions not represented in (8.3) are provided by the higher-order system (6.21). We use these infrastructure to construct solutions to binary interactions of planar waves in the phase space and show that the Jacobian of the inverse of the hodograph transform does not vanish, so we obtain in particular a global solution to the gas expansion problem with detailed shapes and positions of the vacuum boundaries and characteristics. 
The invariant regions in the phase space revealed in the process have more potential than what has been used here. For example, we will use them to handle binary interactions of simple waves, which will lead to the eventual construction of global solutions to some fourwave Riemann problems that will not have vacuum in their data, see a forthcoming paper [16].

We have made a comparison of the pair (8.1)(8.2) to the pair of eigenvalue $\xi=\lambda(u)$ and wave curve system $\left(\lambda-f^{\prime}(u)\right) u^{\prime}=0$ for the one-dimensional system $u_{t}+f(u)_{x}=0$ from Lax [9]. The wave curves of the one-dimensional case correspond to surfaces in the phase space $(i, u, v)$. It will be a very interesting next step to find out the phase space structure that involves subsonic domains and shock waves as well as the hyperbolic surfaces.

\section{Acknowledgement}

J. Li thanks the Department of Mathematics at Penn State University, and Y. Zheng thanks the mathematics department at Capital Normal University for the hospitality during their mutual visits when this work was done. Both authors appreciate discussions with Tong Zhang.

\section{References}

[1] G. Ben-dor and I. I. Glass, Domains and boundaries of non-stationary oblique shock wave reflection, J. Fluid Mech., (1), 92, (1979), pp. 459-496; (2), 96, (1980), pp. 735756.

[2] T. Chang, G. Q. Chen and S. L. Yang, On the 2-D Riemann problem for the compressible Euler equations, I. Interaction of shock waves and rarefaction waves, Disc. Cont. Dyna. Syst., 1, no. 4, (1995), pp. 555-584.

[3] T. Chang, X. Ji, J. Li, P. Zhang, Y. Zheng, An insidious shock formation in a rarefactive Riemann problem for 2-D compressible Euler equations, preprint, 2004, submitted for publication.

[4] S. X. Chen, Z. P. Xin and H. C. Yin, Global shock waves for the supersonic flow past a perturbed cone, Comm. Math. Phys., 228, No. 1, (2002), 47-84.

[5] R. Courant and K. O. Friedrichs, Supersonic flow and shock waves, Interscience Pulishers, Inc., New York, 1948.

[6] C. Dafermos, Hyperbolic Conservation Laws in Continuum Physics (Grundlehren der mathematischen Wissenschaften), pp.443, Springer, 2000

[7] Z. Dai and T. Zhang, Existence of a global smooth solution for a degenerate Goursat problem of gas dynamics, Arch. Ration. Mech. Anal.. 155 (2000), 277-298.

[8] H. M. Glaz, P. Colella, I. I. Glass, R.L. Deschambault, A numerical study of oblique shock-wave reflections with experimental comparisons, Proceedings of the Royal Society of London, Series A, Mathematical and Physical Sciences, 398(1985), 117-140. 
[9] P. Lax, Hyperbolic systems of conservation laws II, Communications on Pure and Applied Mathematics, Vol. X (1957), 537-566.

[10] P. Lax and X. Liu, Solutions of two-dimensional Riemann problem of gas dynamics by positive schemes, SIAM J. Sci. Compt., 19, no. 2, (1998), pp. 319-340.

[11] L. E. Levine, The expansion of a wedge of gas into a vacuum, Proc. Camb. Phil. Soc., 64, (1968), pp. 1151-1163.

[12] J. Li, Global Solution of an Initial-value Problem for Two-dimensional Compressible Euler Equations, Journal of Differential Equations, Vol. 179, No. 1, 178- 194, 2002

[13] J. Li, On the two-dimensional gas expansion for compressible Euler equations, SIAM J. Appl. Math., 62(2001), 831-852.

[14] J. Li, T. Zhang and S. Yang, The two-dimensional Riemann problem in gas dynamics,Pitman monographs and surveys in pure and applied mathematics 98, Addison Wesley Longman limited, 1998.

[15] J. Li, T. Zhang and Y. Zheng, Simple waves and a characteristic decomposition of the two dimensional compressible Euler equations, Coтmu Math Phys, 267 (2006), 1-12.

[16] J. Li and Y. Zheng, Global solutions to some two-dimensional Riemann problems for the compressible isentropic Euler equation, work in preparation.

[17] T. Li, Global classical solutions for quasilinear hyperbolic systems, John Wiley and Sons, 1994.

[18] T. Li and W. Yu, Boundary value problem for quasilinear hyperbolic systems, Duke University, (1985).

[19] A. G. Mackie, Two-dimensional quasi-stationary flows in gas dynamics, Proc. Camb. Phil. Soc., 64, (1968), pp. 1099-1108.

[20] A. Majda and E. Thomann, Multi-Dimensional shock fronts for second order wave equations, Comm. PDE., 12 (7) (1987), 777-828.

[21] I. A. Pogodin, V. A. Suchkov and N. N. Ianenko, On the traveling waves of gas dynamic equations, J. App.. Math. Mech., 22, (1958), pp. 256-267.

[22] C. W. Schulz-Rinne, J. P. Collins and H. M. Glaz, Numerical solution of the Riemann problem for two-dimensional gas dynamics, SIAM J. Sci. Compt., 4, no. 6, (1993), pp. $1394-1414$.

[23] J. Smoller, Shock waves and reaction-diffusion equations, 2nd ed., Springer-Verlag, (1994).

[24] V. A. Suchkov, Flow into a vacuum along an oblique wall, J. Appl. Math. Mech., 27 (1963), pp. 1132-1134.

[25] R. Wang and Z. Wu, On mixed initial boundary value problem for quasilinear hyperbolic system of partial differential equations in two independent variables (in Chinese), Acta Scientiarum, Naturalium of Jinlin University, (1963), pp. 459-502.

[26] Tong Zhang and Yuxi Zheng, Conjecture on the structure of solution of the Riemann problem for two-dimensional gas dynamics systems, SIAM J. Math. Anal., 21 (1990), 593-630.

[27] Yuxi Zheng, Systems of Conservation Laws: Two-Dimensional Riemann Problems, 38 PNLDE, Birkhäuser, Boston, 2001. 